Research Article

\title{
Performance Evaluation of Different Control Methods for an Underactuated Quadrotor Unmanned Aerial Vehicle (QUAV) with Position Estimator and Disturbance Observer
}

\author{
Ghulam E. Mustafa Abro ${ }^{(\mathbb{D},},{ }^{1,2,3}$ Zain Anwar Ali ${ }^{(\mathbb{D}},{ }^{3}$ Saiful A. Zulkifli, ${ }^{1}$ \\ and Vijanth Sagayan Asirvadam (1) ${ }^{1}$ \\ ${ }^{1}$ Department of Electrical and Electronic Engineering, Universiti Teknologi PETRONAS, Seri Iskandar, 32610 Perak, Malaysia \\ ${ }^{2}$ Condition Monitoring Systems Lab, NCRAI Mehran University of Engineering and Technology, Sindh, Pakistan \\ ${ }^{3}$ Sir Syed University of Engineering and Technology, Gulshan E Iqbal, Karachi, Pakistan
}

Correspondence should be addressed to Ghulam E. Mustafa Abro; ghulam.engr@gmail.com

Received 28 August 2021; Revised 29 October 2021; Accepted 16 November 2021; Published 7 December 2021

Academic Editor: Adnan Maqsood

Copyright (C) 2021 Ghulam E. Mustafa Abro et al. This is an open access article distributed under the Creative Commons Attribution License, which permits unrestricted use, distribution, and reproduction in any medium, provided the original work is properly cited.

\begin{abstract}
The main aim of this manuscript is to design and demonstrate the performance of different control algorithms with position estimator and disturbance observer to track the helical trajectory by an underactuated quadrotor craft under the influence of unmodelled dynamic factors and external disturbances. The manuscript consists of the derivations related to the kinematics and dynamics of quadrotor dully derived using the Newton Euler approach. It is one of the strenuous tasks to stabilize and control the quadrotor for helical trajectory tracking since it is an underactuated mechatronic system. In addition to this, with inclusion of unmodelled dynamic factors, it faces some of the serious transient and steady-state issues including Zeno noise. In this research manuscript, dual-loop single-dimension fuzzy sliding mode control (DLSDF-SMC) is proposed to improve the helical trajectory tracking performance, and to tackle the unmodelled dynamic factors, a state feedback controller is proposed consisting of a position estimator and disturbance observer design. The entire system is distributed into two subsystems such that within the angular subsystem, the attitude control is proposed using DLSDF-SMC, and for the translational subsystem, the paper proposes the position control design based on the hyperbolic function to avoid the gimbal lock issue. The overall stability of the proposed closed-loop control scheme is also proved. The simulation work for the proposed algorithm is performed using MATLAB and Simulink software and compared with the conventional sliding mode control (SMC) and fuzzy-based SMC control designs. This work demonstrates that the DLSDF-SMC control technique with position estimator and disturbance observer design in feedback not only improves the aggressive maneuvers while tracking the helical trajectory but also tackles the transient and steadystate issues.
\end{abstract}

\section{Introduction}

The quadrotor unmanned aerial vehicle (QUAV) has four control inputs and six degrees of freedom (DOF). Since the number of control inputs is less than the DOF, that is, $\operatorname{rank}(F(q))<\operatorname{dim}(q)$, where $F(q)$ is the input matrix and $q$ is the state variables. This shows that the external forces are unable to exert the change in acceleration in an arbitrary direction in $q$; therefore, it is known as an underactuated system. Such mechatronic systems are very difficult to stabilize because of unmodelled dynamic factors and external disturbances [1-4]. Controlling and stabilizing, QUAV is one of the strenuous tasks, but if controlled properly with an intelligent control design, then it has several maneuvering capabilities to perform, that is, hovering in the air, path following, and vertical take-off and landing [5-9]. These quadrotor UAVs are used commonly in various domains [10]. Researchers have proposed a number of control strategies such that classical proportional integral derivative (PID) control for regulating [11] and evaluating the transient and steady-state factors [12]. Later, one may also see some of the robust and adaptive control designs to 
improvise these issues [13]. For the Cartesian position, researchers proposed several robust control designs such as backstepping control design [14]. All these control techniques were proposed to ensure better tracking performance, but, still there are some of the limitations observed, and this is all because of the impact of unmodelled dynamic factors [15]. Therefore, researchers proposed some of the adaptive control designs such that neural network-based control design that entertains the sudden changes that occur during the flight of quadrotor through online framework and have certain capabilities of tackling non-linear uncertainties [16].

In literature, one may find the utilization of adaptive schemes such that fuzzy logic control (FLC) for similar issues [17], but due to sub-steps such as fuzzification, inference, and defuzzification, the quadrotor is unable to stimulate aggressive maneuvers. The trend of amalgamating more than one control design is also noticed. Researchers have hybridized the FLC with neural network for robust tracking performance but encounter deviation due to unmodelled dynamic factors and external disturbances [18]. One may notice several adaptive schemes as proposed in [19] where researchers tried to tackle the impact of external disturbances and unnecessary time delays in accelerations [20] using a robust attitude controller along with a state feedback scheme. Some of these algorithms are also proposed for hypersonic vehicles and unmanned aerial vehicles to compensate for the effect of unmodelled dynamic factors and time-varying uncertainties [21,22]. So far, all algorithms are proposed in an ideal or controlled environment where they have either upper or lower bounds of uncertainties. As per the best knowledge of the authors, the practical scenario is quite different from this controlled scenario, thus; very few researchers have focused on factors such as chattering noise that is a high number of oscillations experienced by the four rotors of an underactuated quadrotor during an aggressive maneuver. Researchers have focused either on transient and steady-state issues or the chattering phenomenon during an aggressive maneuver under unmodelled dynamic factors at one time [4]. The term robust control designs itself indicates that they are proposed for bounded values, that is, sliding mode control (SMC), which is proposed mostly for nonlinear mechatronic systems. This is because of the insensitiveness to such external disturbances and factors on the manifold [23-29].

Discussing the issues such that the strong dynamic coupling and singularity issue (gimbal lock), there are several research contributions that have proposed disturbance observers and estimators [30], state estimation using fuzzy scheme [31], approximation using neural network [32,33], and extended state observer [34]. Summarizing these schemes, one may find difficulty in tuning the parameters of disturbance observer, the low convergence rate for the neural network. This is one of the major reasons that people opted for extended state observer (ESO) because of its convergence rate over finite time index. Thus, the paper proposes an easy way to design the disturbance observer (DO) along with the position estimator to produce efficient results with less complexity for tuning the parameters for
DO. If DO is studied properly, one may find it very easy to design as it requires less information about the model and has an ability to tackle the dynamic coupling with uncertainties over a large variation scale $[35,36]$. In terms of the research contribution, the paper provides a detailed study over the tracking issues with an inclusion of unmodelled dynamic factors. These factors are changing instantly; therefore, a novel control approach is amalgamated with a feedback control design that is divided into two sub-blocks, that is, position estimator and disturbance observer to estimate these bounded but instant changes. In addition to this, the reader may see the comparative analysis of the proposed technique with the conventional techniques. Mathematical proof is also provided to prove the stability analysis of the proposed control algorithm along with the proof that ensures the easy tuning of gains of observer design to achieve the faster convergence.

Moreover, the manuscript is divided into six sections. One may find the basic introduction in Section 1, explaining the background about previous control and estimation techniques. Section 2 describes the state-of-the-art approaches specifically and discusses the main limitations on which the solution in this paper is proposed. The mathematical derivation is well explained in Section 3, whereas Section 4 is all about control designs. This begins with the details of simple SMC, FSMC, and design related to dualloop single-dimension fuzzy sliding mode control. Last but not the least, the discussion for position estimator and disturbance observer design is also included within this section. One can also find the stability proof for the proposed control algorithm within this section. In Section 5, the comparative results in terms of simulations in between three control algorithms, namely, SMC, FSMC, and DLSDF-SMC based on position estimator and disturbance observer designs are discussed. Finally, Section 6 presents a comprehensive conclusion for the entire research contribution.

\section{Research Background}

Studying some of the basic control designs such as proportional integral derivative (PID), sliding mode control (SMC), and linear quadratic regulator (LQR), it is noticed that SMC resolves some of the performance issues as compared to conventional LQR and PID control designs [36]. The only limitation with the SMC design is the high number of oscillations that appear on four rotors known as the chattering or Zeno effect [36]. To reduce this effect, one may hybridize the conventional SMC design with single-dimension fuzzy logic control (FLC) [37]. With such hybridization, it does not only reduce these oscillations but also enables an underactuated quadrotor unmanned aerial vehicle (QUAV) for aggressive maneuvering. By studying this approach thoroughly, one may identify a slow convergence rate. This is because of the instant variations in unmodelled dynamic factors. Therefore, this paper proposes the amalgamation of position estimator and disturbance observer to not only observe these changes but also estimate them to derive a faster convergence rate $[18,38]$. In this research paper, the authors derive a Newton-Euler dynamic model with hyperbolic function to address the singularity issue. 
Moreover, it suggests a dual-loop configuration of single-dimension-based fuzzy sliding mode control (DLSDF-SMC). This control design will be controlling and stabilizing the attitude and altitude of the quadrotor. However, the DO and PE will estimate the variations and provide significant feedback to control design. Once the feedback is received, the tuning parameters of single-dimension fuzzy sliding mode control will be tuned accordingly. This results in getting a faster convergence rate and stable helical trajectory tracking. In our work, the position estimator and disturbance observer observe and estimate the variations to tune the parameters of the DLSDFSMC control design. Similar techniques have been used for air robots for path following with pigeon inspired optimization algorithm (PIO) [39-41] instead of simple position estimator and disturbance observer. The main purpose of introducing $\mathrm{DO}$ and $\mathrm{PE}$ here is to optimize the weighting matrix $Q$ in SDFSMC that will ease the design.

This paper proposes dual-loop single-dimension fuzzy sliding model control design for helical trajectory tracking using position estimator and disturbance observer design because sliding mode control is insensitive to unmodelled dynamic factors, but it generates high oscillations [42]. To reduce this oscillatory effect, one may find the fusion of SMC with fuzzy logic control (FLC) [43]. This fusion is done to reduce the Zeno/chattering effect, but it consumes high power as well as processing time, which is one of the limitations of this technique. The reason for consuming high power and processing time is because of the intermediate steps of FLC, that are, fuzzification, inferences of rules, and defuzzification to compute the centre of gravity (CoG) [38]. There are some intelligent and hybrid versions of control that are proposed in [48], but they are inappropriate for underactuated quadrotor craft because of real-time tuning ability, and in most cases, they derive an impulsive behaviour [44]. A similar type of scheme is fuzzy $H_{\infty}$ output feedback control that produces chattering effect and time delay due to the impulsive behaviour [45-49]. Looking at the current literature, one may see the actuator disturbance rejection method [74]. This is presented to improve the overall attitude tracking performance, but in the end, one may notice the delay in accelerations. One may also see the issue of uncertainty is addressed by proposing a non-linear dynamic inversion estimator design $[75,76]$. At the time of introducing disturbance, the quadrotor may experience some chattering effect and may deviate from helical trajectory as well. Regarding the observer design, one must consider the dynamics of the brushless DC motor into account because this will increase the observer bandwidth and will ensure more stability [77]. Studying all about the control and observer designs, this paper presents the current state of the art approaches along with their main constraints in the next sub-section.

2.1. State of the Art Approaches. In this sub-section, the paper discusses the current state-of-the-art approaches that have been successful so far to address the tracking issues in the presence of unmodelled dynamic factors [18]. In the proposed work [18], researchers utilized the perks of disturbance observer (DO) to estimate the changes in unmodelled dynamic factors such as wind disturbance, payload variation, and the loss of rotor's effectiveness. In this strategy, one may see two sub-control systems based on proportional integral (PI) that are responsible for compensating the positional errors whereas backstepping control design is the second one. This second sub-control design tackles the velocity error rates. The study states that there are some time delays in theoretical and predicted accelerations while an increment in thrust from 2,450 RPM to 3,320 revolution per minute (RPM). Moreover, there is a steady-state error of $2 \%$ in the presence of a payload mass of 200 grams. To tackle these issues, research proposed the inclusion of an integral term denoted as $C_{i i}=0.5$ that resolves the issues but again produces some of the serious overshoots when the payload is released by quadrotor at any specific location. Discussing the case of rotor's effectiveness loss, there is the same behaviour observed as it was in the case of payload variation. One may find the different versions of SMC that are either hybridized or used in different configurations such that dual-loop integral SMC (DLI-SMC) [48] with LESO. After critical analysis of theoretical results, one may see that the kinematics and dynamics of an underactuated quadrotor proposed in the paper have not considered the uncertainties. LESO used with this technique estimates the smooth changes so far. Thus, this paper suggests the use of DO and position estimator along with the hyperbolic function to tackle not only the smooth as well as nonsmooth variations but also the issues such as chattering effect as well as steady-state error. In [48], one cannot understand the significant utilization of integral terms proposed with SMC in this dual-loop configuration. The second state-of-theart approach is named as predictive optimal control design with the hybridization of disturbance observer (DO) [49]. The proposed strategy really works for smooth and non-smooth variations during its flight with a payload mass of 200 grams. In addition to this, one can see POC performs robust within the interval of $\pm 25 \%$ of perturbations only. Thus, the performance of the proposed technique in [49] needs some more experimental work to be performed, whereas the disturbance observer (DO) whose parameters are normally very difficult to tune seems easy to adjust and tune. Thus, this paper adapts the same knowledge related to disturbance observers only from this research work.

Third-order sliding mode control (TOSMC) is also one of the current approaches to address the issues of the chattering effect [50]. Here quaternion model is proposed to avoid the issues like gimbal lock. Still, one may observe the chattering effect that never reduced even after using highand low-order filters. These are the current trending approaches, and their limitations are summarized in Table 1.

There are few articles that have considered the factors such as input saturation that most of the cases lead to the complexity of the control algorithm [51]. To address these constraints, researchers used the global sliding surface based on trajectory tracking error. For a fast convergence rate, researchers have proposed the virtual control input with an integral version, but this can bring some of the serious overshoots in case of payload variation. One may see the supertwisting version of sliding mode control (ST-SMC) 
TABLE 1: State-of-the-art approaches with limitations.

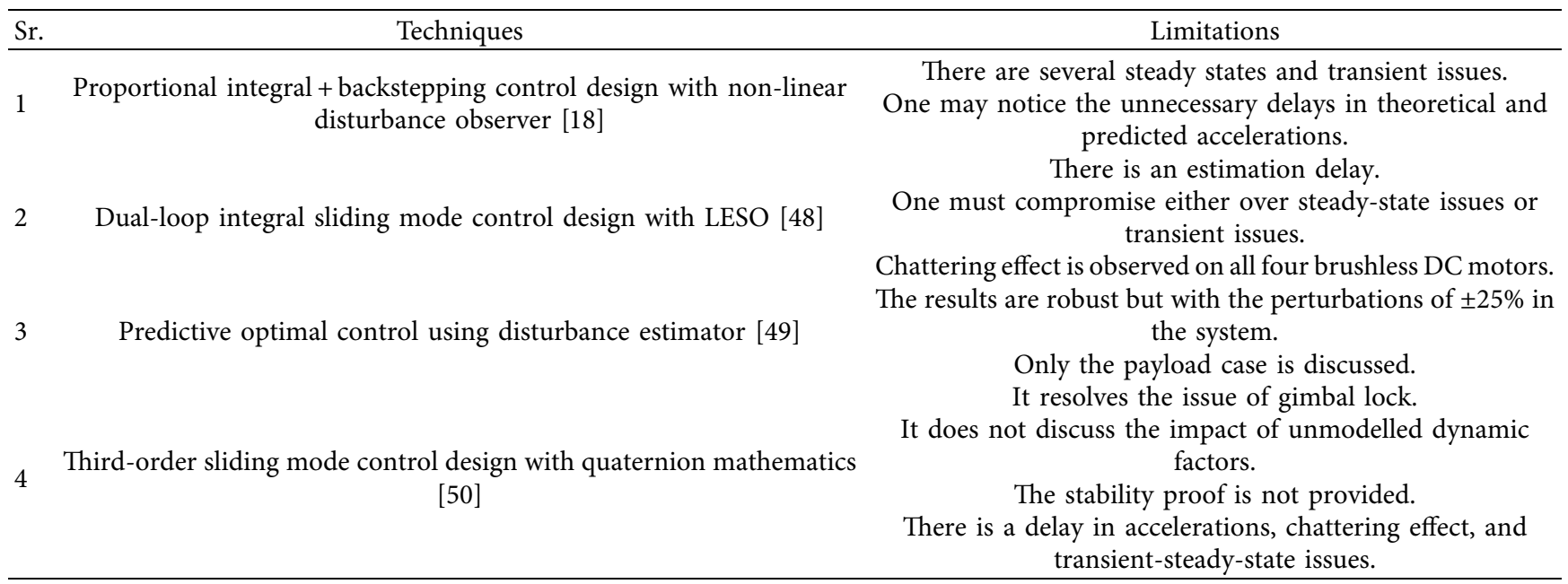

that is proposed to achieve the finite time position and attitude stability for an underactuated quadrotor craft. The simulation results related to input delayand other factors such as wind disturbance and model uncertainty are addressed. The only limitation with this algorithm can be seen in the figures where the researchers mentioned the time histories of supertwisting switching surfaces. This can be one of the reasons for high oscillations known as Zeno phenomena over the four brushless DC (BLDC) motors later [52]. Researchers have proposed proportional integral derivative control with sliding mode control (SMC) with an adaptive flavour to ensure stability but if one may see the time responses of the control inputs even under known bounded disturbance, there is a chattering effect in the inputs [53].

Moreover, the measured values obtained from several contributions are stated in Table 2 to convey the main performance factors that must be in mind while performing theoretical or simulation solutions.

Table 2 shows the seven significant performance factors that have been addressed by several researchers, but still, there is a need to improvise them. This is the only reason that the design of a control system for an underactuated quadrotor unmanned aerial vehicle (QUAV) is still one of the challenging problems in the presence of unmodelled dynamic factors. There is a need for an intelligent control design along with a predictive observer technique that can optimize the entire dynamic system to have fast, accurate, and stabilized trajectory tracking with better performance factors as indicated in Table 2.

2.2. Research Contribution. The single-dimension-based fuzzy sliding mode control in the simple configuration without any estimator and observer design has been proposed for the stability of various applications such that NPS autonomous vehicle [54], double pendulum-type overhead cranes [55], DSRV that stands for deep submergence rescue vehicle, and many other systems [56]. After studying the several control designs $[57,58]$ one may conclude that sliding mode control provides better tracking performance when amalgamated with fuzzy logic control (FLC) [59]. By adding fuzzy mathematics, there is a slow convergence rate observed. This is because of intermediate steps of fuzzification, inference, and defuzzification. Thus, the manuscript proposes a novel dual-loop singledimension-based fuzzy sliding mode control with a position estimator and disturbance observer to tackle the unmodelled dynamic factors and lumped uncertainties during the tracking of helical trajectory. In addition to this, the proposed algorithm enables our underactuated quadrotor craft to perform aggressive maneuvers. The main research contributions are mentioned as follows:

(i) The tracking issues are addressed in a better way than [60-62] along with an inclusion of unmodelled dynamic factors. In addition to this, the software simulations with different additional trajectory tracking prove the robustness of the proposed algorithm.

(ii) In the paper, the instant changes are observed and estimated using a feedback control that comprises a position estimator and disturbance observer, unlike the other proposed observers that estimate the bounded variations only [63-65].

(iii) In contrast with other control designs discussed in $[13,66]$, the proposed dual-loop single-dimensionbased fuzzy sliding mode control is an asymptotic stable, and one may see the stability proof of the proposed closed-loop control design provided within the paper.

(iv) Disturbance observer and position estimator proposed in this paper enable tuning of parameters for faster convergences so that the tracking errors may converge to zero as soon as possible.

With the above research postulates, the proposed control strategy is robust, and one of its kinds, it surely improves the performance of all parameters as discussed in Table 2.

\section{Mathematical Model}

The main part to design single-dimension-based fuzzy sliding mode control begins with the proposition of a mathematical model for underactuated quadrotor craft. 
TABLE 2: Measured versus required performance factors.

\begin{tabular}{lccc}
\hline Sr. & Performance factors & Measured performance & Required performance \\
\hline 1 & Steady-state error & $2 \%-5 \%$ & $1 \%-2 \%$ \\
2 & Settling time & 15.0 & $<15.0$ seconds \\
3 & Overshoots & $10 \%-15 \%$ & $<10 \%$ \\
4 & State estimation delay & 19.5 seconds & $<19.5$ seconds \\
5 & Zeno/chattering effect & $2.35-3.75 \%$ & $<2.375 \%$ \\
6 & Tuning of disturbance observer & Difficult & Easy or moderate \\
7 & Delay in accelerations & 17.5 & $<17.5$ seconds \\
\hline
\end{tabular}

Thus, the coordinate transformation is discussed in this section. One may see the quadrotor in real time as a mechatronic system comprises four fixed rotors mounted at four ends of a cross frame as shown in Figure 1(a). In most cases, these rotors are propelled by using brushless DC (BLDC) motors. This is because their revolution per minute (RPM) can easily be changed using an electronic speed controller (ESC). As we know, we have two types of coordinate systems such that body-fixed coordinate systems illustrated as $o x y z$ and ground-fixed coordinate systems OXYZ. This is illustrated in Figure 1(b).

In Figure 1(b), one may see $\phi, \theta, \psi$ are roll, pitch, and yaw, respectively. These three angles are mainly controlled via four control input forces such as $F_{1}, F_{2}, F_{3}, F_{4}$. These forces can be changed by varying the RPMs of four BLDC motors. The weight of underactuated quadrotor craft is given as $m g$, where $m$ is the mass of quadrotor and $g$ is the acceleration due to gravity. One may opt the lifting forces and torques from the body-fixed coordinate system as illustrated oxyz. However, one may have to understand the exertion of these forces in the groundfixed frame $O X Y Z$. Thus, the transformation can be done as shown in Figure 2.

One can obtain the transform matrix of each axis from oxyz to $O X Y Z$ as follows:

$$
\begin{aligned}
& R(x, \phi)=\left[\begin{array}{ccc}
1 & 0 & 0 \\
0 & c \phi & -s \phi \\
0 & s \phi & c \phi
\end{array}\right], \\
& R(y, \theta)=\left[\begin{array}{ccc}
c \theta & 0 & s \theta \\
0 & 1 & 0 \\
-s \theta & 0 & c \theta
\end{array}\right], \\
& R(z, \psi)=\left[\begin{array}{ccc}
c \psi & -s \psi & 0 \\
s \psi & c \psi & 0 \\
0 & 0 & 1
\end{array}\right],
\end{aligned}
$$

where the symbol $c$ and $s$ are cosine and sine functions. One may use the following equation to have interconversion between body-fixed coordinate (oxyz) and ground-fixed coordinate $(O X Y Z)$ :

$$
R(\phi, \theta, \psi)=R(z, \psi) R(y, \theta) R(x, \phi) .
$$

To simplify the model of the quadrotor, there are some assumptions to be followed as mentioned below: (i) The physical structure of the quadrotor prototype is considered as one of the rigid and symmetrical structures, and the body-fixed coordinate origin must coincide with the centre of mass.

(ii) There is a proportional relationship between torque and DC voltages. Moreover, one may ignore the air resistance and gyroscopic effect, while the quadrotor is flying at low speed.

(iii) Consider that the attitude change is very small (i.e., less than $5^{\circ}$ ).

The lifting forces are derived in body-fixed coordinate systems and are labelled as $F_{1}, F_{2}, F_{3}$ and $F_{4}$. One may derive the value of net force as follows:

$$
F_{B}=\left[\begin{array}{c}
F_{x} \\
F_{y} \\
F_{z}
\end{array}\right]=\left[\begin{array}{c}
0 \\
0 \\
F_{1}+F_{2}+F_{3}+F_{4}
\end{array}\right] .
$$

The interconversion can be done using the following equation:

$$
F_{G}=\left[\begin{array}{c}
F_{x} \\
F_{y} \\
F_{z}
\end{array}\right]=R(\phi, \theta, \psi) \cdot F_{B}=\left(\sum_{i=1}^{4} F_{i}\right)\left[\begin{array}{c}
c \phi s \theta c \psi+s \phi s \psi \\
c \phi s \theta s \psi-s \phi c \psi \\
c \theta c \phi
\end{array}\right] .
$$

As per the equation of motion, one may try to deduce the following equations:

$$
\begin{aligned}
& \ddot{x}=\frac{F_{X}}{m}, \\
& \ddot{y}=\frac{F_{y}}{m}, \\
& \ddot{z}=\frac{F_{z}}{m}-g .
\end{aligned}
$$

Similarly, one may deduce the attitude change from the Newton-Euler formula as follows:

$$
\mathfrak{\Im} \dot{\omega}+\omega \times \mathfrak{\Im} \omega=\tau,
$$

where the term $\mathfrak{I}$ is defined as $\operatorname{diag}\left(\mathfrak{J}_{\theta}, \mathfrak{J}_{\phi}, \mathfrak{J}_{\psi}\right)$ and $\mathfrak{J}_{\theta}, \mathfrak{J}_{\phi}, \mathfrak{\Im}_{\psi}$ and are equivalent, respectively, with the moment of inertia about the axis for pitch, roll, and yaw. In addition to this, the term $\omega=(\dot{\theta}, \dot{\phi}, \dot{\psi})^{T}$, whereas the notation for torque is denoted as $\tau=\left(\tau_{1}, \tau_{2}, \tau_{3}\right)^{T}$. This torque term consists of two types of torques such that $M_{i}$ 


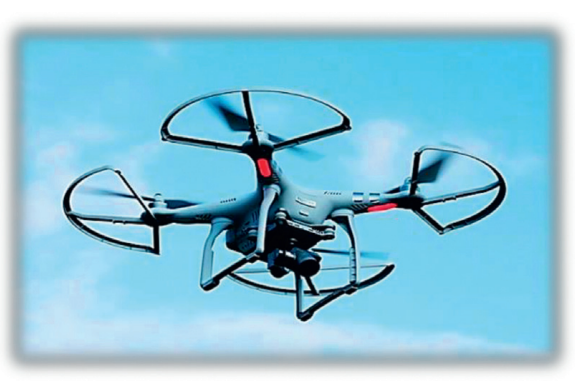

(a)

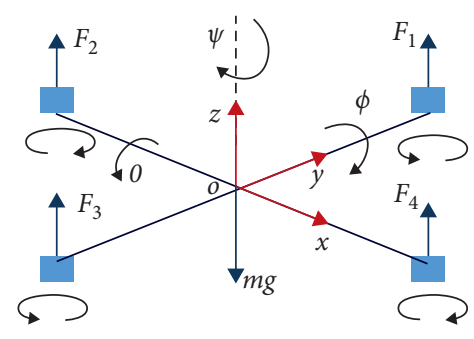

(b)

FIGURE 1: (a) Underactuated quadrotor physical model and (b) body-fixed coordinate.

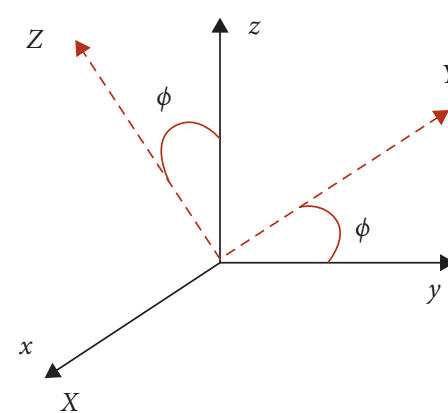

(a)

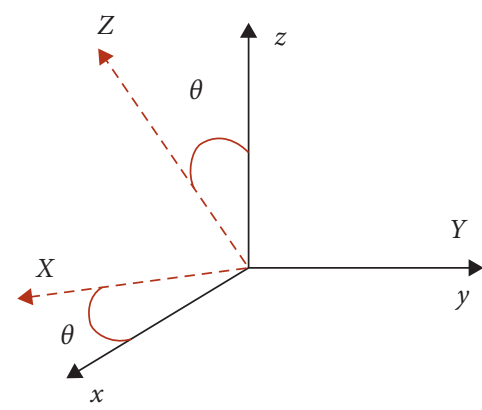

(b)

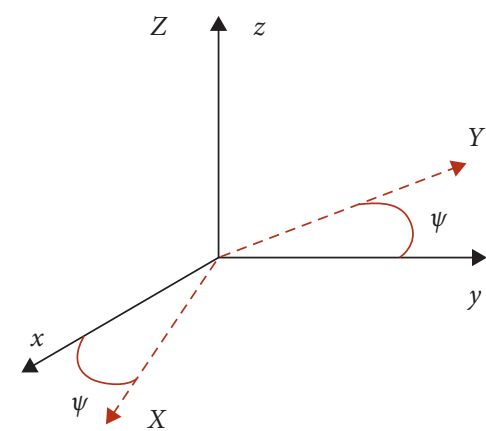

(c)

FIgURe 2: Roll, pitch, and yaw angles: (a) roll, (b) pitch, and (c) yaw.

that is the turning effect generated by the lifting forces and $\tau_{i}^{1}$ is the torque generated by self-rotation of the rotors of BLDC motors. The relation can be seen in the following equation:

$$
\tau=\left(\begin{array}{c}
\tau_{1} \\
\tau_{2} \\
\tau_{3}
\end{array}\right)=\left(\begin{array}{c}
\tau_{1}^{1} \\
\tau_{2}^{1} \\
\tau_{3}^{1}
\end{array}\right)+\left(\begin{array}{c}
M_{1} \\
M_{2} \\
M_{3}
\end{array}\right), \quad \text { whereas }\left\{\begin{array}{l}
\tau_{1}^{1}=\mathfrak{\Im}_{\gamma} \Omega \dot{\phi}, \\
\tau_{2}^{1}=\mathfrak{\Im}_{\gamma} \Omega \dot{\theta}, \\
\tau_{3}^{1}=\mathfrak{J}_{\gamma} \dot{\Omega} .
\end{array}\right.
$$

The torque generated by the lifting forces is as follows:

$$
\begin{aligned}
& M_{1}=l\left(F_{3}-F_{1}\right), M_{2}=l\left(F_{4}-F_{2}\right), \\
& M_{3}=F_{2}+F_{4}-F_{1}-F_{3} .
\end{aligned}
$$

Moreover, the value of omega is as follows:

$$
\Omega=\Omega_{1}-\Omega_{2}+\Omega_{3}-\Omega_{4} .
$$

Studying equations (10) and (11), one may derive the following equation:

$$
\left\{\begin{array}{l}
\mathfrak{J}_{\gamma} \Omega \ddot{\phi}=\left[\dot{\theta} \dot{\psi}\left(\mathfrak{\Im}_{\phi}-\mathfrak{\Im}_{\psi}\right)+\mathfrak{J}_{\gamma} \dot{\theta} \Omega+M_{2}\right], \\
\mathfrak{J}_{\gamma} \Omega \ddot{\theta}=\left[\dot{\phi} \dot{\psi}\left(\mathfrak{J}_{\psi}-\mathfrak{\Im}_{\theta}\right)+\mathfrak{J}_{\gamma} \dot{\phi} \Omega+M_{1}\right], \\
\mathfrak{J}_{\gamma} \Omega \ddot{\psi}=\left[\dot{\phi} \dot{\theta}\left(\mathfrak{J}_{\theta}-\mathfrak{J}_{\phi}\right)+\mathfrak{J}_{\gamma} \dot{\Omega}+\Omega\right] .
\end{array}\right.
$$

The input variables of the underactuated quadrotor unmanned aerial vehicle (QUAV) is defined as follows:

$$
\left\{\begin{array}{l}
U_{1}=F_{1}+F_{2}+F_{3}+F_{4}=K_{t}\left(\Omega_{1}^{2}+\Omega_{2}^{2}+\Omega_{3}^{2}+\Omega_{4}^{2}\right), \\
U_{2}=F_{4}-F_{2}=K_{t}\left(\Omega_{4}^{2}-\Omega_{2}^{2}\right), \\
U_{3}=F_{3}-F_{1}=K_{t}\left(\Omega_{3}^{2}-\Omega_{1}^{2}\right), \\
U_{4}=-\Omega=K_{d}\left(-\Omega_{1}^{2}+\Omega_{2}^{2}-\Omega_{3}^{2}+\Omega_{4}^{2}\right),
\end{array}\right.
$$

where $U_{1}$ is the main control variable for vertical speed and the control variable of roll angle is denoted by $U_{2}$. Pitch angle is controlled using the control variable of $U_{3}$, whereas the control variable of $U_{4}$ is denoted for yaw angle. Furthermore, the variable $\Omega_{i}$ is the rotating speed, where $i=1,2,3$ and 4 denoting the number of rotors. Lastly, the coefficients of lifting force and torques are given as $K_{t}$ and $K_{d}$, respectively. 
Studying all equations from (6) to (15), the mathematical model for our proposed underactuated quadrotor is presented as follows:

$$
\left\{\begin{array}{l}
\ddot{x}=\frac{(\cos \phi \sin \theta \cos \psi+\sin \phi \sin \psi) U_{1}}{m}, \\
\ddot{y}=\frac{(\cos \phi \sin \theta \sin \psi-\sin \phi \cos \psi) U_{1}}{m}, \\
\ddot{z}=\frac{(\cos \theta \cos \phi) U_{1}-g,}{m} \\
\ddot{\phi}=\frac{\left[\dot{\theta} \dot{\psi}\left(\mathfrak{\Im}_{\phi}-\mathfrak{\Im}_{\psi}\right)+\mathfrak{\Im}_{\gamma} \dot{\theta} \Omega+l U_{2}\right]}{\mathfrak{J}_{\theta}}, \\
\ddot{\theta}=\frac{\left[\dot{\phi} \dot{\psi}\left(\mathfrak{\Im}_{\psi}-\mathfrak{\Im}_{\theta}\right)+\mathfrak{\Im}_{\gamma} \dot{\phi} \Omega+l U_{3}\right]}{\mathfrak{\Im}_{\phi}}, \\
\ddot{\psi}=\frac{\left[\dot{\phi} \dot{\theta}\left(\mathfrak{\mho}_{\theta}-\mathfrak{\Im}_{\phi}\right)+\mathfrak{J}_{\gamma} \dot{\Omega}+U_{4}\right]}{\mathfrak{\Im}_{\psi}},
\end{array}\right.
$$

where the notation $\mathfrak{\Im}_{\gamma}$ is the equivalent moment of inertia

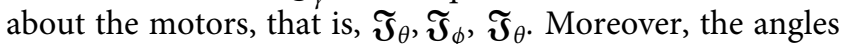
such that roll, pitch, and yaw are denoted as $\phi, \theta$, and $\psi$, respectively. The notation $l$ is the distance between pivot to each BLDC motors (rotors) such that $\ddot{x}, \ddot{y}$ and $\ddot{z}$ are accelerations about each axis. The term $U_{i}$ is the equivalent input variable, the sum of the four rotors' rotating speed is given by $\Omega$, where the weight of proposed underactuated quadrotor is denoted by $m$ and acceleration due to gravity is mentioned as $g$. These all parameters along with their definition are summarized in Table 3.

Opting the variables as $X=(x 1, x 2, x 3, x 4, x 5, x 6)^{T}=$ $(\phi, \dot{\phi}, \theta, \dot{\theta}, \psi, \dot{\psi})^{T}$, whereas the input for the entire dynamic system is $U=\left(U_{1}, U_{2}, U_{3}, U_{4}\right)^{T}$. Thus, equation (16) can be transformed as follows:

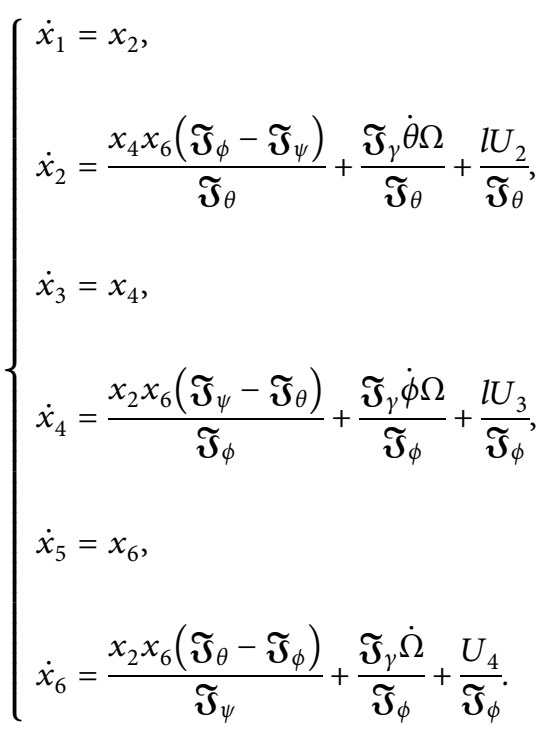

For proposing the above-mentioned dynamic model, there are some assumptions to be considered as mentioned below:

(i) During flight mode, the accelerations in the bodyfixed coordinate are approximately equal to the acceleration in the ground-fixed coordinate

(ii) However, the issue related to cross-coupling effects of angular speeds and gyroscopic effects are negligible. Thus, the state-space model can be derived using the standard definition as follows:

$$
\begin{aligned}
& X=A X+B U, \\
& Y=C X
\end{aligned}
$$

$$
A=\left[\begin{array}{llllll}
0 & 1 & 0 & 0 & 0 & 0 \\
0 & 0 & 0 & 0 & 0 & 0 \\
0 & 0 & 0 & 1 & 0 & 0 \\
0 & 0 & 0 & 0 & 0 & 0 \\
0 & 0 & 0 & 0 & 0 & 1 \\
0 & 0 & 0 & 0 & 0 & 0
\end{array}\right],
$$$$
B=\left[\begin{array}{ccc}
0 & 0 & 0 \\
\frac{l}{\mathfrak{\Im}_{\theta}} & 0 & 0 \\
0 & 0 & 0 \\
0 & \frac{l}{\mathfrak{\Im}_{\theta}} & 0 \\
0 & 0 & 0 \\
0 & 0 & \frac{l}{\mathfrak{\Im}_{\theta}}
\end{array}\right] \text {, }
$$

$$
C=\left[\begin{array}{llllll}
1 & 0 & 0 & 0 & 0 & 0 \\
0 & 1 & 0 & 0 & 0 & 0 \\
0 & 0 & 1 & 0 & 0 & 0 \\
0 & 0 & 0 & 1 & 0 & 0 \\
0 & 0 & 0 & 0 & 1 & 0 \\
0 & 0 & 0 & 0 & 0 & 1
\end{array}\right] .
$$

The values computed for $\left(l / \mathfrak{\Im}_{\theta}\right),\left(l / \mathfrak{\Im}_{\phi}\right),\left(l / \mathfrak{\Im}_{\psi}\right)$ are $3.834,3.834$, and 8.334 , respectively.

\section{Control Design}

This section discusses the design steps for dual-loop singledimension-based fuzzy sliding mode control design along with an amalgamation of PE and DO. This proposed algorithm will make sure the better performance of an underactuated quadrotor craft while tracking the helical trajectory in the presence of external disturbances and unmodelled dynamic factors. Equation (16) shows all six control variables that are dully controlled using four control 
TABLE 3: Summary of all parameters for the dynamics of underactuated quadrotor craft.

\begin{tabular}{lcc}
\hline Definition & Parameter & Value \\
\hline Distance between pivot and each BLDC motors & $l(\mathrm{~m})$ & 0.250 \\
Equivalent inertia about the pitch axis & $J_{\theta}\left(\mathrm{kg} \cdot \mathrm{m}^{2}\right)$ & 0.0652 \\
Equivalent inertia about the roll axis & $J_{\phi}\left(\mathrm{kg} \cdot \mathrm{m}^{2}\right)$ & 0.0652 \\
Equivalent inertia about the yaw axis & $J_{\psi}\left(\mathrm{kg} \cdot \mathrm{m}^{2}\right)$ & 0.120 \\
Coefficient of torques & $K_{d}\left(\mathrm{~N} \cdot \mathrm{m} \cdot \mathrm{s}^{2}\right)$ & 0.00748 \\
Coefficient of lifting forces & $K_{t}\left(\mathrm{~N} \cdot \mathrm{s}^{2}\right)$ & 0.0221 \\
Weight of quadrotor & $m(\mathrm{~kg})$ & 2.75 \\
Acceleration due to gravity & $\mathrm{msec}^{-2}$ & 9.8 \\
\hline
\end{tabular}

inputs such as $U_{1}, U_{2}, U_{3}, U_{4}$. To design the algorithm, this section has been divided into further sub-sections for the readers to understand better.

4.1. Dual-Loop Single-Dimension-Based Fuzzy Sliding Mode Control. This sub-section discusses the theory about sliding mode control design and its hybrid version single input based fuzzy sliding mode control design. The basic block diagram of sliding mode control SMC is illustrated in Figure 3. This control design comprises a steering infinite quadrotor's trajectory as a sliding manifold. This is designed to lead the underactuated quadrotor UAV coming all the way from any position to target to follow a specific trajectory and converge the states asymptotically to zero.

The sliding surface for conventional sliding mode control design is as follows:

$$
s_{f}=\dot{e}-c e .
$$

where the term $s_{f}$ is known as sliding surface, where the error rate is denoted by $e$ and the rate of change of error rate is indicated as $\dot{e}$. The gain is shown as $c$ that can be tuned as per the requirement of the controller. Moreover, the derivative of the sliding surface is as follows:

$$
\dot{s_{f}}=-\rho s_{f}-k \operatorname{sign}\left(s_{f}\right) \text {, }
$$

where the positive diagonal definite matrices are denoted as $\rho$ and $k$. There are several research manuscripts where scholars provided the hybrid versions of SMC by amalgamating it with fuzzy logic control (FLC) design. This type of hybridization had been proposed to overcome the constraints, that is, Zeno or chattering effect that may damage the propellers and brushless DC (BLDC) motors [44,68]. This hybridization not only reduces the chattering but also enables the underactuated quadrotor to perform extraordinary aggressive maneuvers. The sliding surface depends on variables such as $e$ and $\dot{e}$ that can be computed using equations (21) and (22). The main purpose of fuzzy logic is to tune these two variables.

$$
s_{f}=\left[\begin{array}{ll}
e & \dot{e}
\end{array}\right]\left[\begin{array}{l}
k \\
1
\end{array}\right] \text {. }
$$

To design FLC, one should design the rule base system. In this manuscript, the rules are proposed with the help of vectoral distances between state trajectory $\left(L_{s n}\right)$ and manifold $\left(L_{o}\right)$. Mathematically, it is possible to use the error and rate of change error as illustrated in the following equations:

$$
\begin{gathered}
L_{s n}=\frac{\left(\dot{e_{\mathrm{Q}}}+k \dot{e_{\mathrm{Q}}}\right)}{\sqrt{1+k^{2}}}, \\
L_{o}=\sqrt{N^{2}-L_{s n}^{2}} .
\end{gathered}
$$

This derivation is well illustrated in Figure 4, whereas the rules for fuzzy-based sliding mode control (FSMC) are defined in Table 4.

The membership function type opted is the triangular type and is illustrated in Figure 5. The output of FLC shall be singleton type based on the centre of gravity ( $\mathrm{CoG})$ as shown in Figure 6. Moreover, the scale defined for all input and output membership functions varies between \pm 0.6 .

The diagram shown in Figure 7 is as same as the block diagram for a conventional SMC design illustrated in Figure 3. There is an addition of fuzzy logic control (FLC) and the sub-block shown in the red-dashed box. In this subblock, the vectoral distance is computed and delivered later to FSMC Block to actuate the drone with the desired behaviour.

This manuscript focuses on main issues such that tackling the unmodelled dynamic factors using position estimator and disturbance observer design. The reader may observe that the estimated values that are feedback to this block where it will tune the parameters for dual-loop singledimension-based fuzzy sliding mode control (DLSDFSMC). By doing this, DLSDF-SMC not only reduces the computation time but also has a faster convergence rate while tracking the trajectory from any position in the $3 \mathrm{D}$ plane. A single-dimension-based version of FSMC is proposed because of several perks that a simple FSMC cannot provide such as fast convergence rate with aggressive maneuvers. This is because of intermediate sub-processes such that fuzzification, inference of two-dimensional rules, and defuzzification for producing CoG-based output. Therefore, converting the $2 \mathrm{D}$ table for rules into a single dimension using the signed distance method $[37,69]$ can be one of the methods to improve the convergence rate and obtain the aggressive maneuvering options. This can be possible and shown graphically in Figure 8.

After studying the graphical illustration in Figure 9, one may derive the mathematical relationship for the $G$ variable as follows: 


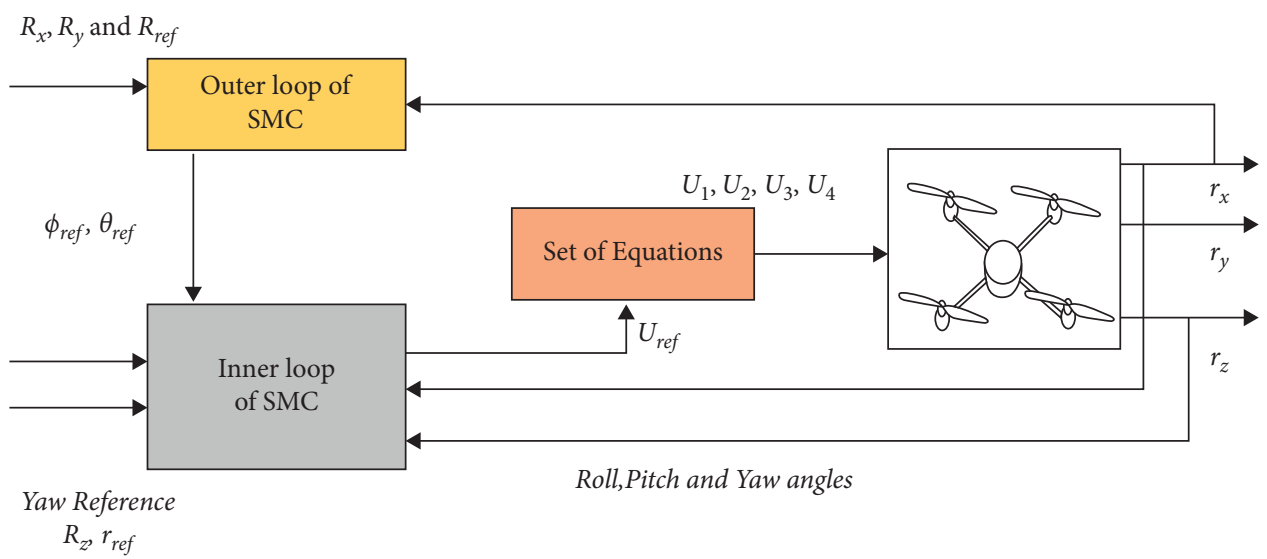

FIGURE 3: Block diagram for sliding mode control design.

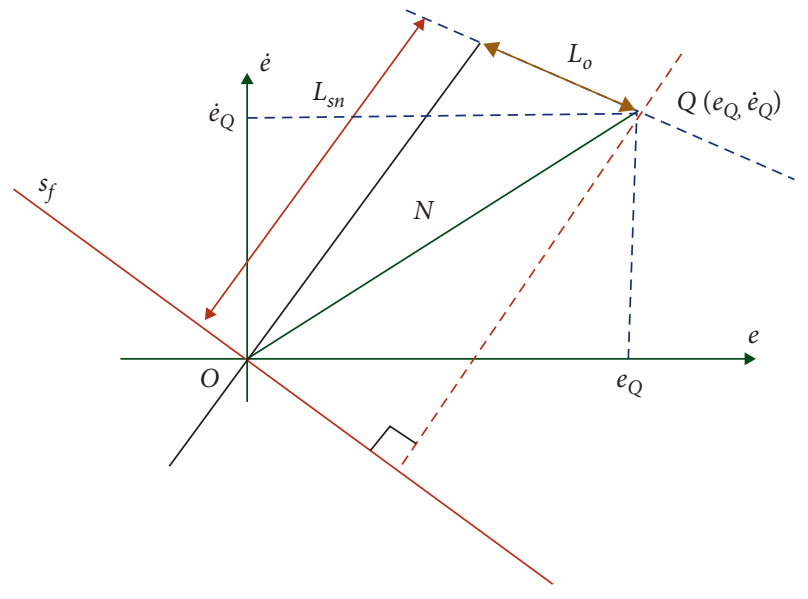

FIgURE 4: Vectoral illustration for $L_{s n}$ and $L_{o}$.

TABLE 4: Rules based on vectoral distance.

\begin{tabular}{lccccccc}
\hline$L_{o}$ & & & \multicolumn{5}{c}{$L_{s n}$} \\
& NB & NS & NS & Z & PS & NS & PB \\
\hline PB & Z & PS & PM & PB & PB & PB & PB \\
NS & NS & Z & PS & PM & PB & PB & PB \\
PS & NM & NS & Z & PS & PM & PB & PB \\
Z & NB & NM & NS & Z & PS & PM & PB \\
NS & NB & NB & NM & NS & Z & PS & PM \\
NS & NB & NB & NB & NM & NS & Z & PS \\
NB & NB & NB & NB & NB & NB & NS & Z \\
\hline
\end{tabular}

PB: positive big, PS: positive small, NB: negative big, NS: negative small, PM: positive medium, Z: zero, and NM: negative medium.

$$
\begin{aligned}
& L_{s n}+\alpha L_{o}=0, \\
& G=\frac{L_{s n}+\alpha L_{o}}{\sqrt{1+\alpha^{2}}},
\end{aligned}
$$

where the variable $G$ is a diagonal vector derived from Table 4 as illustrated in Figure 9.

Table 5 is reproduced using equations (26) and (27). This time, the input membership functions are triangular type but with different $x$ - index values, whereas the output

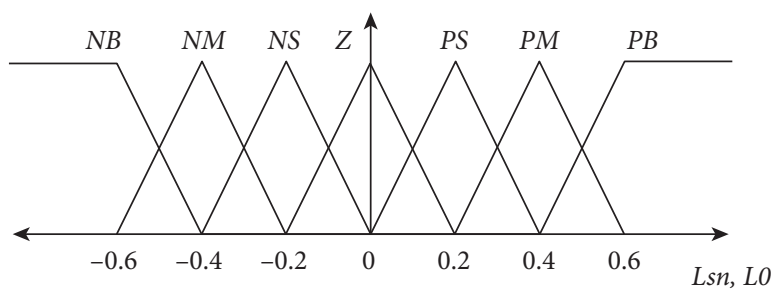

FIgURE 5: Triangular-type input membership functions.

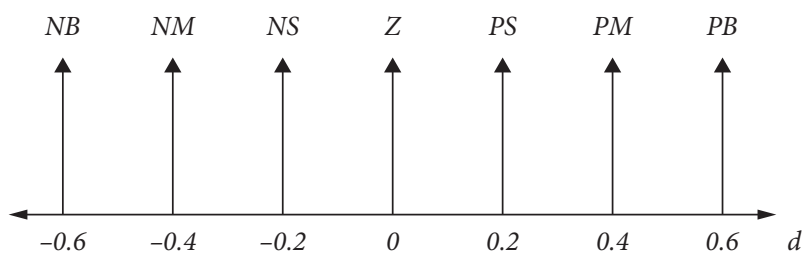

FIgURE 6: Singleton-type output membership function based on CoG.

membership function will remain the same as discussed and shown in Figures 5 and 6. Having this entire information, one may now start designing the linear extended state observer.

4.2. Position Estimator and Disturbance Observer. To tackle the unmodelled dynamic factors, it is essential to propose a fine feedback structure comprised a position estimator and disturbance observer. This is possible by the deployment of various sensors to underactuated quadrotor craft. The most frequent sensors are inertial measurement unit (IMU), GPS, a computer vision system, optical flow sensor for the position alignment, and lastly the utilization of an ultrasonic or infrared sensor, that is, LiDAR v3 for altitude. While considering the data from these sensors, they all must be preprocessed to avoid the noise and extract only useful data [70]. This position estimator is designed to compute and measure the signals that cannot join several noisy signals to remove the noise. As the presence of such random noise can lead towards the deterioration of the final position with time for our proposed underactuated 


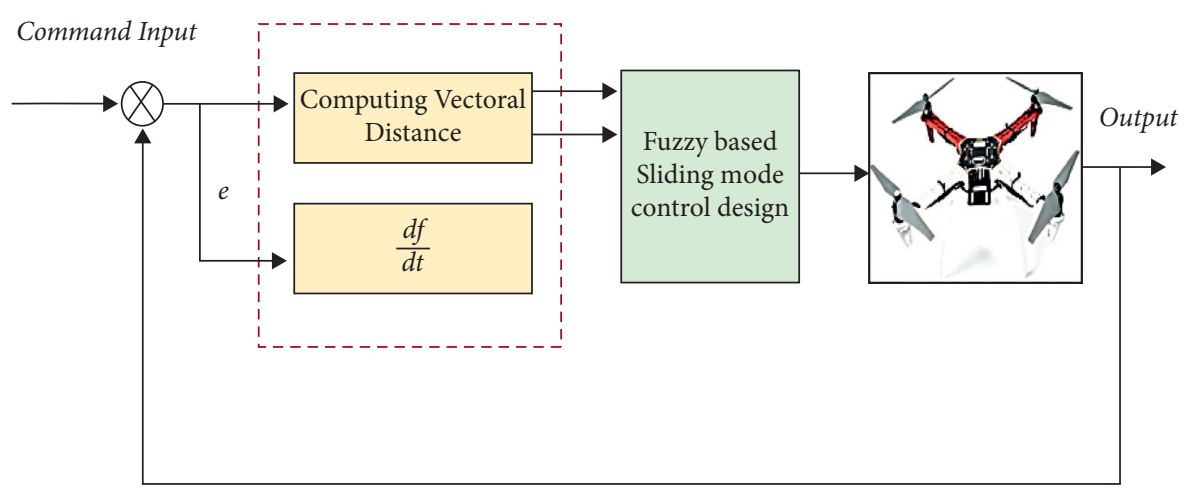

Figure 7: Block diagram of fuzzy-based SMC design.

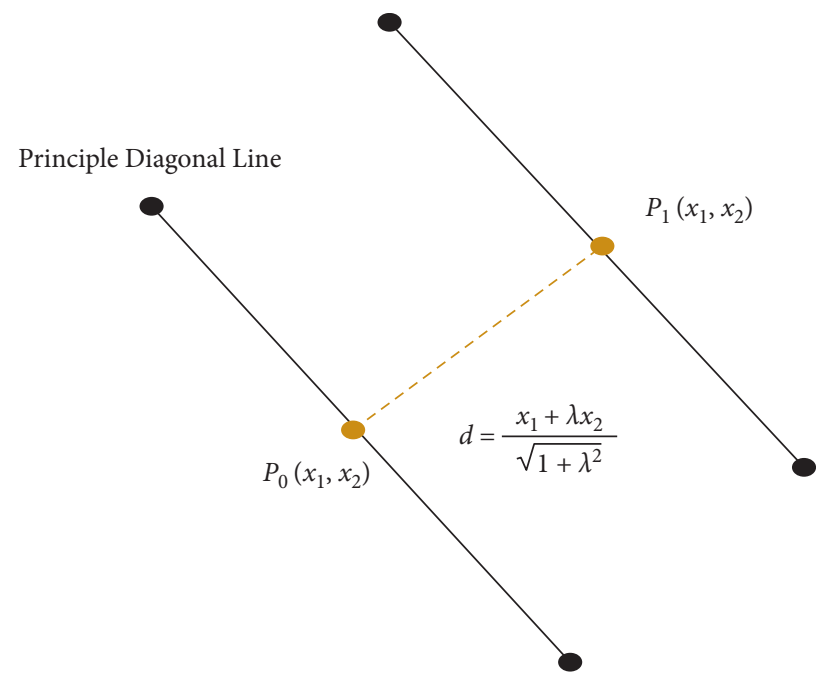

FIGURE 8: Graphical representation to derive $G$ variable.

\begin{tabular}{|c|c|c|c|c|c|c|c|}
\hline & NB & NS & NS & Z & PS & NS & PB \\
\hline $\mathrm{PB}$ & Z & PS & PM & PB & $\mathrm{PB}$ & $\mathrm{PB}$ & PB \\
\hline NS & NS & $z^{\prime}$ & PS & PM & 'PB & $\mathrm{PB}$ & PB \\
\hline PS & NM & NS & $\checkmark z$ & PS & PM & $\mathrm{PB}$ & PB \\
\hline Z & NB & NM & NSS & Z & PS, & PM & $\mathrm{PB}$ \\
\hline NS & NB & NB & NM & 'NSS & $Z$ & PS & PेM \\
\hline NS & NB & NB & 'NB & NM & 'NSS & 'Z् & PS \\
\hline NB & NB & NB & NB & $\mathbb{N B}$ & NB & NSS & $z_{\text {}}$ \\
\hline
\end{tabular}

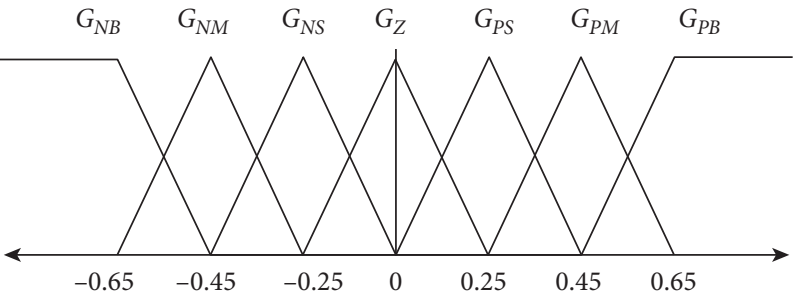

Figure 9: Deriving single-dimension input membership functions using the diagonal variable $G$.

TABLe 5: Single-dimension rules using $G$ variable.

\begin{tabular}{llcccccc}
\hline$G$ & $G_{\mathrm{NB}}$ & $G_{\mathrm{NM}}$ & $G_{\mathrm{NS}}$ & $G_{\mathrm{Z}}$ & $G_{\mathrm{PS}}$ & $G_{\mathrm{PM}}$ & $G_{\mathrm{PS}}$ \\
\hline$G^{\prime}$ & $\mathrm{NB}$ & $\mathrm{NM}$ & $\mathrm{NS}$ & $\mathrm{Z}$ & $\mathrm{PS}$ & $\mathrm{PM}$ & PB \\
\hline
\end{tabular}


quadrotor unmanned aerial vehicle (QUAV). In the presence of unmodelled dynamic factors such as wind disturbance, our proposed disturbance observer ensures the performance of position tracking and the linear velocities by reducing the noise and effect of external disturbances. Discussing the position estimator, it computes the positional and velocity vectors for quadrotor UAV. These measure signals via IMU may contain an amplified noise while acquiring the velocity and position vectors. The frequent sensor for acquiring the positional vectors is the GPS sensor module which is commonly working at a $10 \mathrm{~Hz}$ frequency rate that means every new value will appear after every $100 \mathrm{~ms}$. In Figure 10, it has been depicted that the main aim of the estimator is to acquire the data of position and velocity at the frequency rate of $100 \mathrm{~Hz}$.

As per the above diagram, the measured variable is the actual positional and velocity vectors that are acquired from IMU. In addition to this, total thrust is also considered to observe and estimate the linear acceleration via a dynamic model of underactuated quadrotor $\mathrm{UAV}$. The outcome is the linear estimated velocity, which is derived by amalgamating the velocity from estimated linear acceleration and velocity from the positional sensor as shown in Figure 10. The entire calculation is depending on the number of states measured from the signal. In the case of transient issues, the positions are detected by estimated linear acceleration through double integration and rectified at every $100 \mathrm{~ms}$. However, in case of steady-state performance, one has taken the signals directly.

This was all about estimator design, whereas the disturbance observer is implemented to determine the exact amplitude of disturbances caused by unmodelled dynamic factors and external disturbances. Figure 11 presents the detailed working of a disturbance observer that calculates the disturbance as a linear and angular acceleration via the dynamic model of an underactuated quadrotor unmanned aerial vehicle (QUAV). For the synchronization of estimated values with time during the flow of signals from the control block, a delay of motors is included. However, in the case of position, disturbance observer estimation is performed using the states from the accelerometer, and gravity acceleration is reduced using the gravity reduction sub-block as shown in Figure 11.

After studying all the equations, one may derive the observer error of each DO as shown below mathematically:

$$
\begin{aligned}
& \dot{\hat{e}}_{x}=\dot{F}_{1}-\dot{\widehat{F}}_{1}=\left(A_{x}-L_{g x} C_{x}\right) \widehat{e}_{x}+E_{x} \omega_{1}, \\
& \dot{\hat{e}}_{y}=\dot{F}_{2}-\dot{\widehat{F}}_{2}=\left(A_{y}-L_{g y} C_{y}\right) \widehat{e}_{y}+E_{y} \omega_{2}, \\
& \dot{\hat{e}_{z}}=\dot{F}_{3}-\dot{\widehat{F}}_{3}=\left(A_{z}-L_{g z} C_{z}\right) \widehat{e}_{z}+E_{z} \omega_{3}, \\
& \dot{\hat{e}}_{\psi}=\dot{F}_{4}-\dot{\widehat{F}}_{4}=\left(A_{\psi}-L_{g \psi} C_{\psi}\right) \widehat{e}_{\psi}+E_{\psi} \omega_{4} .
\end{aligned}
$$

These equations can be represented in matrix form as follows:

$$
\dot{\hat{e}}=\widehat{H} \widehat{e}+E_{d},
$$

where

$$
\begin{aligned}
& \widehat{e}=\left[\widehat{e}_{x}, \dot{\widehat{e}}_{x}, \ddot{\vec{e}}_{x}, \dddot{\widehat{e}}_{x}, \dddot{\widehat{e}}_{x}, \dot{\vec{e}}_{x}, \widehat{e}_{y}, \dot{\vec{e}}_{y}, \ddot{\widetilde{e}}_{y}, \dddot{\widehat{e}}_{y}, \dddot{\widehat{e}}_{y} \dot{\vec{e}}_{y},\right. \\
& \left.\widehat{e}_{z}, \dot{\hat{e}}_{z}, \ddot{\hat{e}}_{z}, \dddot{\widehat{e}}_{z}, \dddot{\widehat{e}}_{z}, \dot{\hat{\boldsymbol{e}}}_{z}, \widehat{e}_{\psi}, \dot{\hat{\boldsymbol{e}}}_{\psi}, \ddot{\widehat{e}}_{\psi},\right] \text {, } \\
& \widehat{H}=\left[\begin{array}{cccc}
\hat{H}_{1} & O_{5} & O_{5} & O_{3} \\
O_{5} & \widehat{H}_{2} & O_{5} & O_{3} \\
O_{5} & O_{5} & \widehat{H}_{3} & O_{3} \\
O_{5} & O_{5} & O_{4} & \widehat{H}_{4}
\end{array}\right], \\
& E_{d}=\left[0,0,0,0, \omega_{1}, 0,0,0,0, \omega_{2}, 0,0,0,0, \omega_{3}, 0,0, \omega_{4}\right]^{T},
\end{aligned}
$$

where $\widehat{H}$ is the term defined as the roots of the matrix mentioned in equation (34), and it must be on the left halfplane because of the bandwidths, that is, $\Gamma_{x o}, \Gamma_{y o}, \Gamma_{z o}$, and $\Gamma_{\psi o}$ are non-negative in values. These all-estimated error dynamics as defined in equations (28)-(31) are stable in nature provided by Lemma $1[29,31]$.

Lemma 1. In equation (32), $\lim _{t \rightarrow \infty} \widehat{e}(t)$ is considered as bounded if and only if one of the following two conditions are true such that first one. $\tau_{i}<a, i=1,2,3,4$ for all time index $t$, whereas second one $\omega_{i}<b, i=1,2,3,4$ for all time $t$. If in case $\tau_{i}$ has a constant value, that is, $\omega_{i}=0, i=1,2,3,4$, then the estimated error dynamics shall be considered as asymptotically stable.

4.3. Stability of the Proposed Closed-Loop Control Design. The stability of the proposed closed-loop control system is described here within this sub-section. It starts all from state tracking error vector of $x$ - position:

$$
e_{r x}=F_{d 1}-X_{1}
$$

where the term $F_{d 1}=\left[F_{x d}, \dot{F}_{x d}, \ddot{F}_{x d}, \ddot{F}_{x d}\right]^{T}$ and $X=\left[F_{11}\right.$, $\left.F_{12}, F_{13}, F_{14}\right]^{T}$. This leads to equation (37) as follows:

$$
\dot{F}_{d 1}=A_{1} F_{d 1}+B_{1}\left(\dddot{F}_{x d} \cdot \dot{F}_{x d}\right),
$$

where $A_{1}=\left[\begin{array}{llll}0 & 1 & 0 & 0 \\ 0 & 0 & 1 & 0 \\ 0 & 0 & 0 & 1 \\ 0 & 0 & 0 & 0\end{array}\right], B_{1}=\left[\begin{array}{l}0 \\ 0 \\ 0 \\ 1\end{array}\right]$. In this way, the new feedback can be designed that is written in matrix form as follows:

$$
\dot{X}_{1}=A_{1} X_{1}+B_{1}\left(v_{r x}+\tau_{1}\right) .
$$

Using the following equation, one may derive the feedback design as shown in equation (40): 


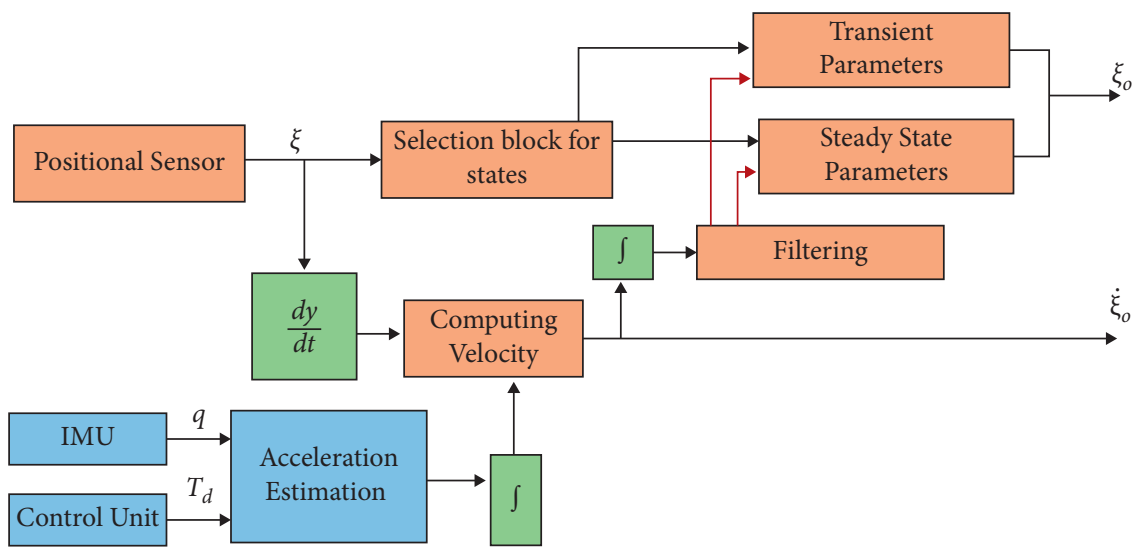

FIGURE 10: Block diagram for proposed position estimator design.

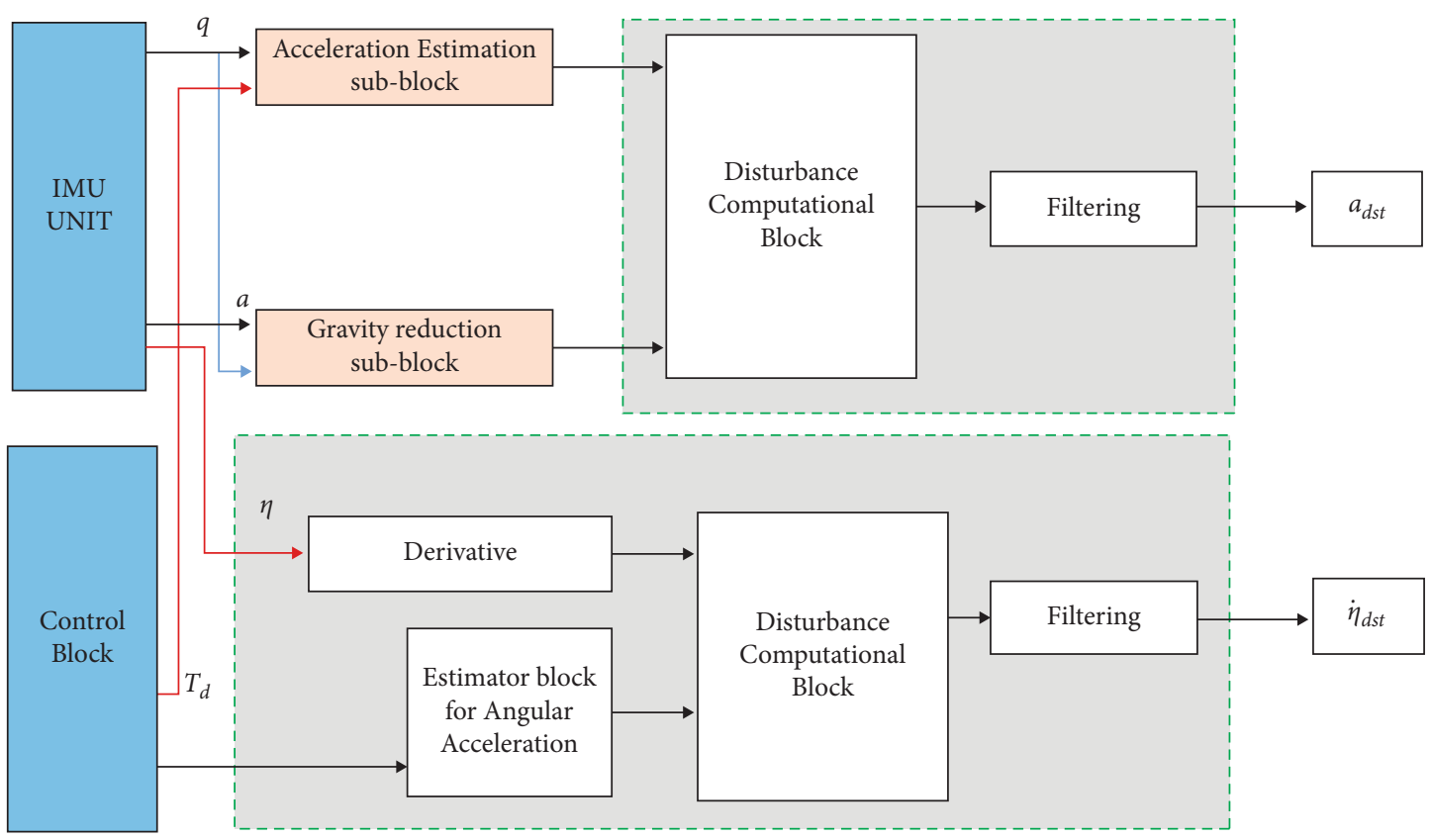

Figure 11: Block diagram of proposed disturbance observer design.

$$
\begin{aligned}
& \widehat{e}_{r 1}=F_{x d}-\widehat{F}_{11}=F_{x d}-F_{11}-\widehat{F}_{11}, \\
& v_{r x}=K_{x}\left(e_{r x}+\widehat{e}_{x}\right)+\left(\stackrel{\mathfrak{t}}{F}_{d x} \cdot \dot{F}_{d x}\right)-\widehat{\tau}_{1} .
\end{aligned}
$$

The variable $K_{x}$ in equation (40) is equal to $\left[K_{x 1}, K_{x 2}, K_{x 3}, K_{x 4}\right]$; thus, the state tracking error dynamics can be expressed more accurately as follows:

$$
\dot{e}_{r 1}=\left(A_{1}-B_{1} K_{x}\right) e_{r x}-B_{1} K_{x} \widehat{e}_{x}-B_{1} \widehat{e}_{x} .
$$

Hence, combining equations (28) and (41), one may obtain

$$
\left[\begin{array}{c}
\dot{e}_{r x} \\
\dot{\hat{e}}_{x}
\end{array}\right]=\left[\begin{array}{cc}
\left(A_{1}-B_{1} K_{x}\right) & -B_{1} K_{x} B_{1} \\
0 & A_{x}-L_{g x} C_{x}
\end{array}\right]\left[\begin{array}{c}
e_{r x} \\
\hat{e}_{x}
\end{array}\right]+\left[\begin{array}{c}
0 \\
E_{x}
\end{array}\right] \omega_{1} .
$$

By using equation (42), one may find the closed-loop stability of the proposed underactuated quadrotor unmanned aerial vehicle (QUAV). This can be possible mathematically if one derives the eigenvalues of the terms, that is, $A_{1}-B_{1} K_{x}$ and $A_{x}-L_{g x} C_{x}$, since the expressions such as $\left(A_{1}, B_{1}\right)$ and $A_{x}, C_{x}$ are controllable and observable. Thus, the stability of such error dynamics for $x$ - position given by equation (42) is ensured by opting for the suitable controller and observer poles. Similarly, the feedback for $y, z$, and $\psi$ can also be designed.

$$
\left[\begin{array}{c}
\dot{e}_{r y} \\
\dot{\hat{e}}_{y}
\end{array}\right]=\left[\begin{array}{cc}
\left(A_{2}-B_{2} K_{y}\right) & -B_{2} K_{y} B_{1} \widehat{e}_{x} \\
0 & A_{x}-L_{g y} C_{y}
\end{array}\right]\left[\begin{array}{c}
e_{r y} \\
\hat{e}_{y}
\end{array}\right]+\left[\begin{array}{c}
0 \\
E_{y}
\end{array}\right] \omega_{2},
$$

$$
\left[\begin{array}{c}
\dot{e}_{r z} \\
\dot{\hat{e}}_{z}
\end{array}\right]=\left[\begin{array}{cc}
\left(A_{3}-B_{3} K_{z}\right) & -B_{3} K_{z} B_{3} \\
0 & A_{z}-L_{g z} C_{z}
\end{array}\right]\left[\begin{array}{c}
e_{r z} \\
\hat{e}_{z}
\end{array}\right]+\left[\begin{array}{c}
0 \\
E_{z}
\end{array}\right] \omega_{3},
$$




$$
\left[\begin{array}{l}
\dot{e}_{r \psi} \\
\dot{\hat{e}}_{\psi}
\end{array}\right]=\left[\begin{array}{cc}
\left(A_{4}-B_{4} K_{\psi}\right) & -B_{4} K_{\psi} B_{4} \\
0 & A_{\psi}-L_{g \psi} C_{\psi}
\end{array}\right]\left[\begin{array}{c}
e_{r \psi} \\
\hat{e}_{\psi}
\end{array}\right]+\left[\begin{array}{c}
0 \\
E_{\psi}
\end{array}\right] \omega_{4} .
$$

Moreover, in the above equations (41)-(45), $A_{1}=A_{2}=A_{3}$, where $B_{1}=B_{2}=B_{3}, K_{y}=\left[K_{y 1}, K_{y 2}, K_{y 3}\right.$, $\left.K_{y 4}\right], K_{z}=\left[K_{z 1}, K_{z 2}, K_{z 3}, K_{z 4}\right]$, and lastly, $K_{\psi}=\left[K_{\psi 1}\right.$, $\left.K_{\psi 2}\right]$. For this work, $A_{4}$ and $B_{4}$ are mentioned as follows:

$$
\begin{aligned}
& A_{4}=\left[\begin{array}{ll}
0 & 1 \\
0 & 0
\end{array}\right], \\
& B_{4}=\left[\begin{array}{l}
0 \\
1
\end{array}\right] .
\end{aligned}
$$

The stability of the closed-loop system $y, z$, and $\psi$ can be obtained in the same way. After studying these all subsections, one may derive the intelligent control algorithm of dual-loop single-dimension fuzzy sliding mode control with position estimator and disturbance observer. The block diagram of the overall control algorithm and system is shown in Figure 12. In this figure, the entire working of dualloop single-dimension fuzzy SMC is summarized. The important factor here is to opt for an appropriate control vector $u(t)$ in a way that the given quadratic performance index mentioned in equation (47). It obtains the minimum value. It is later proved that it reaches the minimum value by means of the proposed control law equation as mentioned in (48).

$$
u(t)=-K X(t)=R^{-1} B^{T} P X(t),
$$

where $P$ is the optimal matrix can be computed using the Riccati equation as follows:

$$
A^{T} P+P A-P B R^{-1} B^{T} P+Q=0 .
$$

In Figure 12, the matrices $Q$ and $R$ are opted to have the form such as $Q=\operatorname{diag}\left(q_{11}, 0, q_{33}, 0, q_{55}, 0\right)$ and $R=\operatorname{diag}(1,1,1,1,1,1)$. The variables such as $q_{11}, q_{33}$ and $q_{55}$ will be compensated using PE and DO propose with dualloop single-dimension fuzzy slide mode control that will take care of the oscillatory response of the system. The implementation of the proposed algorithm follows belowmentioned steps in simple:

(i) Establishing the dynamics for underactuated quadrotor craft

(ii) Tuning the performance index and weighting $Q$ and $R$ matrices accordingly

(iii) Estimating the parameters for the proposed DLSDF-SMC control algorithm using PE and DO as shown in Figure 12

\section{Simulation Results}

In this section, simulation work is carried using MATLAB and Simulink software to show the comparative performance among SMC, FSMC, and dual-loop single-dimension fuzzy sliding mode control technique with a fusion of position estimator and disturbance observer design. The parameters for quadrotor craft used in the below-mentioned simulations are $m=2.75 \mathrm{~kg}, J_{\theta}=0.0652 \mathrm{kgm}^{2}, J_{\phi}=0.0652$ $\mathrm{kgm}^{2}, J_{\psi}=0.120 \mathrm{kgm}^{2}, l=0.25 \mathrm{~m}$ and $g=9.81 \mathrm{~ms}^{-2}$.

5.1. Sliding Mode Control Technique. During the execution of conventional sliding mode control for an underactuated quadrotor craft dynamic model, one observes the deviation at the $66^{\text {th }}$ second, and it remains deviated from the helical trajectory. In Figure 13, one may see the impact of unmodelled dynamic factors.

The unmodelled dynamic factors and unknown external disturbances have been introduced within the Simulink model using a random function generator at the $25^{\text {th }}$ second in all positional vectors that can be seen in Figure 14. This not only affects the positional vectors but also deviates our quadrotor from its desired trajectory. After the inclusion of these factors at the $25^{\text {th }}$ second, one may also notice the high number of oscillations that are known as the chattering or Zeno effect. This is one of the reasons that this paper also proposes to review the hybrid version of SMC with fuzzy logic control (FLC) design.

5.2. Fuzzy-Based Sliding Mode Control. Since there were a high number of oscillations (chattering noise) while executing the SMC Design, the paper thus demonstrates the fusion of fuzzy logic control-based SMC design. One may see in Figure 15, an underactuated quadrotor craft again starts deviating in the presence of unmodelled dynamics factors from its path at the $59^{\text {th }}$ second and coming back again at the $63^{\text {rd }}$ second.

Moreover, this fusion though reduced the chattering effect but also slower the convergence rate. This slow convergence rate is because of the intermediate steps within the fuzzy logic control (FLC) design. One may see the positional response produced through FSMC in Figure 16.

After studying Figure 16, one may see that the number of high oscillations (chattering/Zeno effect) is reduced now in these simulations, but there is still deviation of our proposed underactuated quadrotor UAV with inclusion of unmodelled dynamic factors.

5.3. DLSDF-SMC Control with PE and DO Design. Looking at the constraints such as high computation time, deviation, and slower convergence rate, one may conclude that the proposed UAV will not be able to perform the aggressive maneuvers. Thus, this paper proposes the pigeoninspired optimization-based dual-loop single-dimension SMC control technique that improves the performance not only in terms of positional vectors but also over the limitations of FSMC and conventional SMC. In addition to this, it tackles the unmodelled dynamic factors very smartly. The demonstration for tracking the helical trajectory is shown in Figure 17.

One can see the positional vectors in Figure 18. This time, DLSDF-SMC design has tackled the mentioned issues very smartly. The proposed underactuated quadrotor craft 


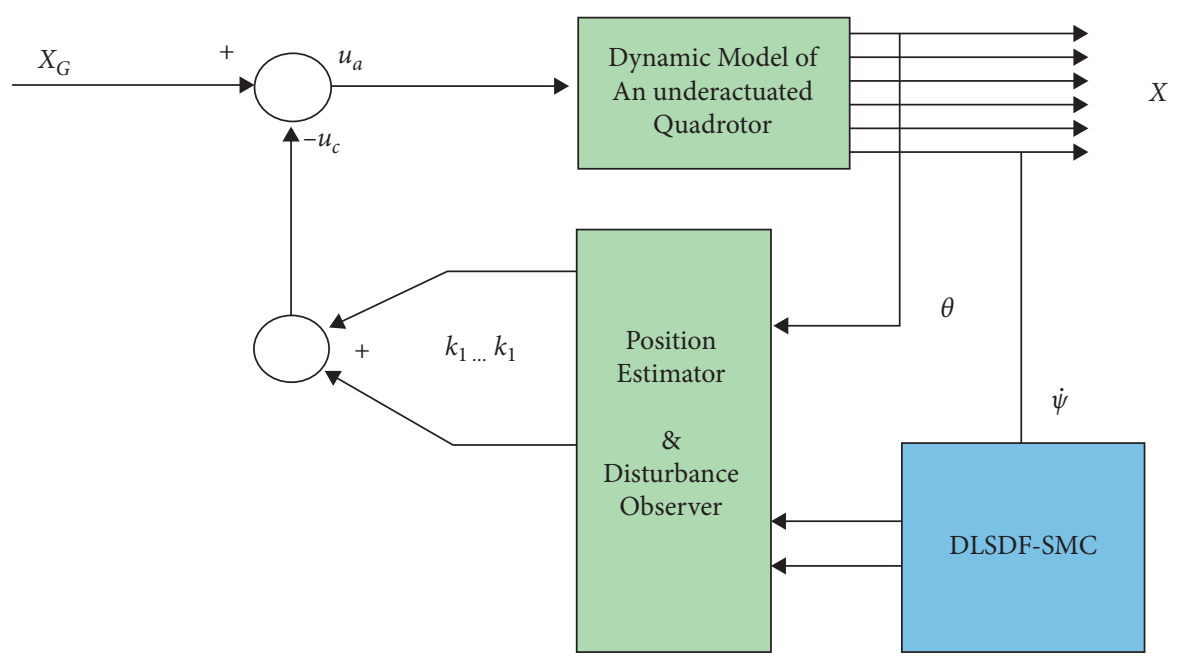

FIGURE 12: Block diagram of dual-loop single-dimension fuzzy SMC with PE and DO.
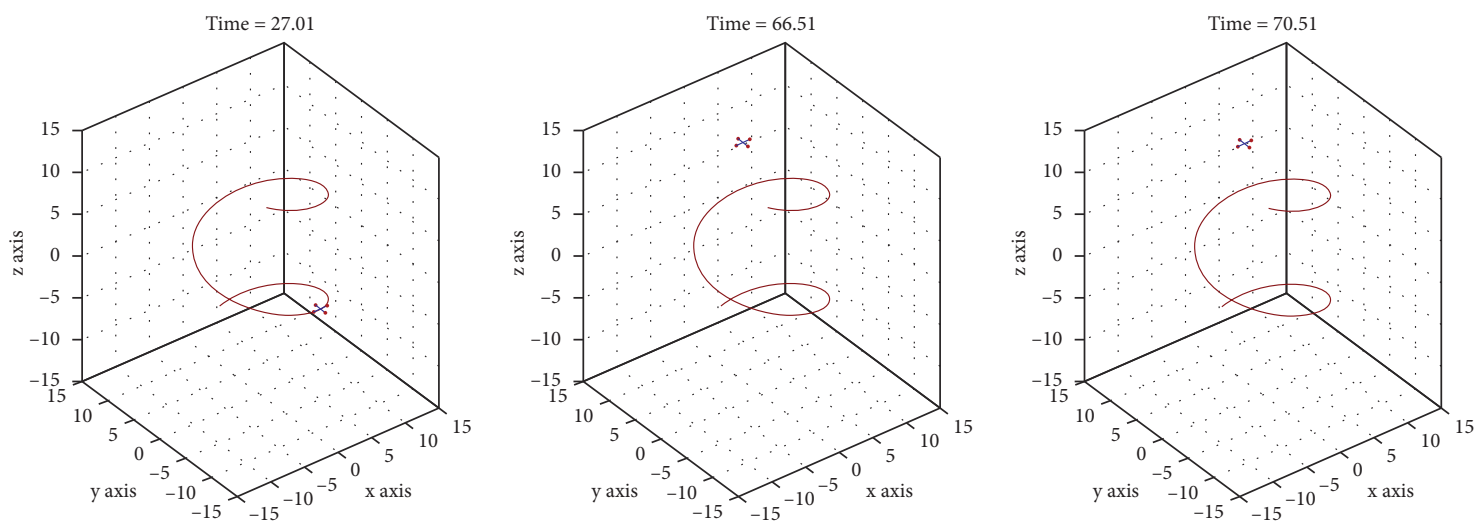

FIGURE 13: Helical trajectory tracking using conventional SMC.

does not deviate from the helical trajectory at any instant with a very low chattering effect.

In these simulation results, there is an improvement in terms of tracking performance. There is no deviation from the helical trajectory and non-observable chattering effect.

\subsubsection{Performance Evaluation for Different Trajectories.} To check the robustness of the proposed algorithm of DLSDF-SMC with PE and DO design, this paper presents two more trajectories tracking along with their responses for positional vectors. One can see this tracking performance illustrated in Figures 19(a) and 19(b). Moreover, their linear velocity response is also illustrated over circular and diamond trajectories in Figures 20 and 21, respectively.

The performance of the proposed fusion of position estimator and disturbance observer is illustrated in Figure 22. In this figure, the real signal is shown in blue; measured signal is shown in green; and finally, the estimated signal is shown in red colour. In the figure, real, measured, and estimated position, and velocity at $z$-axis is shown. Moreover, the attitude disturbance is also estimated and shown in Figure 23. In this figure, the disturbance observer estimates the angular acceleration from desired rotor angular velocities. It should be noted that the measured values are obtained using an inertial measurement unit.

In Figure 23, one may see that the disturbance observer trying to calculate the angular acceleration from angular velocities that are measured using an inertial measurement unit (IMU). The proposed estimating technique based on position estimator and disturbance observer starts estimating at time instant of $20^{\text {th }}$ second and ends it at the $40^{\text {th }}$ second. This is actually a correspondence to an equal force that tries to stabilize the underactuated quadrotor craft while tracking the helical trajectory. In the figure, real, measured, and estimated signals are shown.

Simply, in the above figures, one may see the implementation of DLSDF-SMC with PE and DO technique on two different trajectories. The responses show the algorithm is valid for even circular and diamond trajectories as well. In the diagram, the red line shows the actual trajectory, whereas the blue colour shows the tracking of underactuated quadrotor craft. In addition to this, the entire summary for all three control algorithms is mentioned in Table 6. The comparison is done in terms of few selected factors of concern such that execution time, chattering effect, control input energy, transient, and steady-state values. Among these factors, control input energy has been computed by acquiring the 

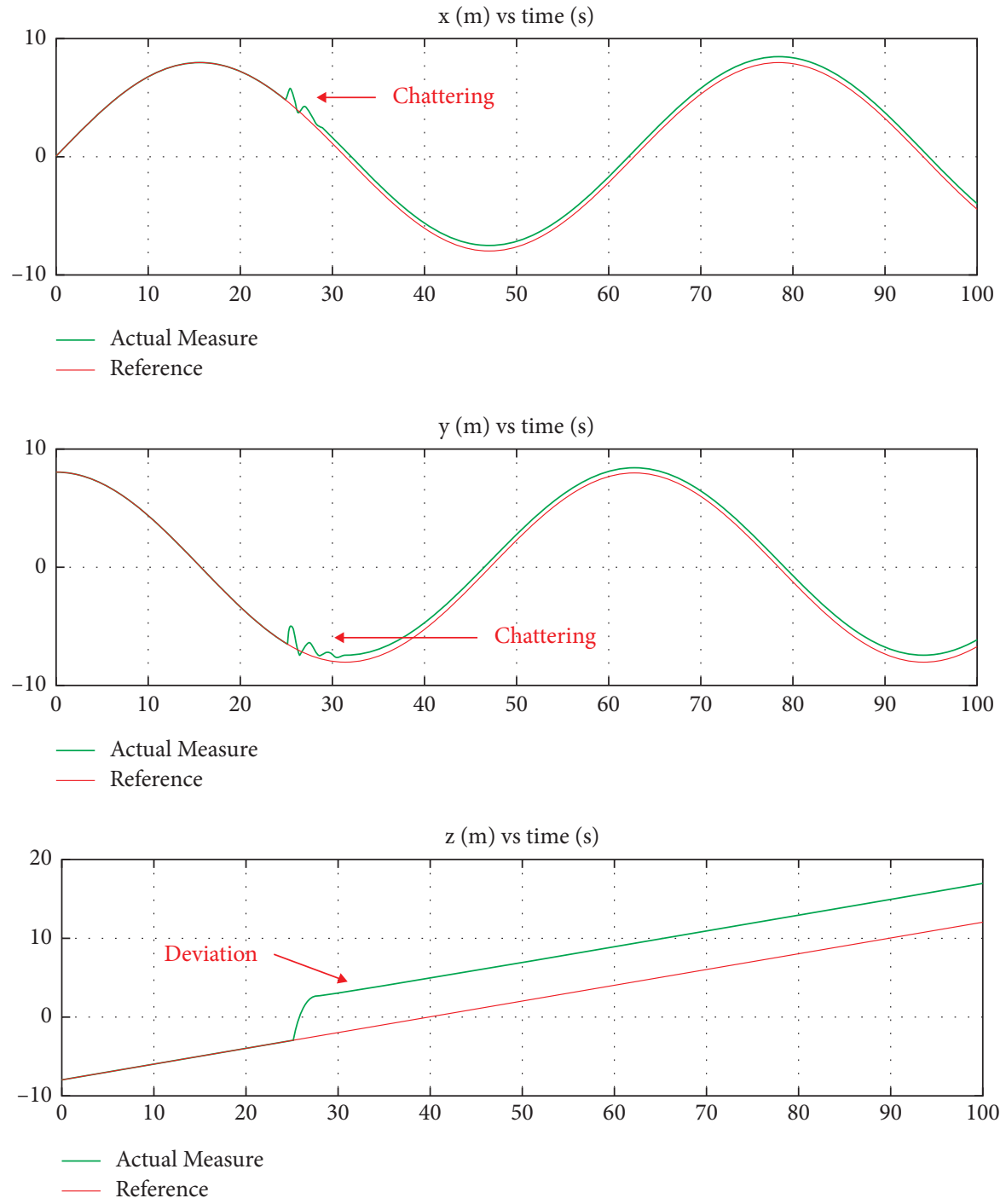

FIGURE 14: Response of positional vectors using conventional SMC design.
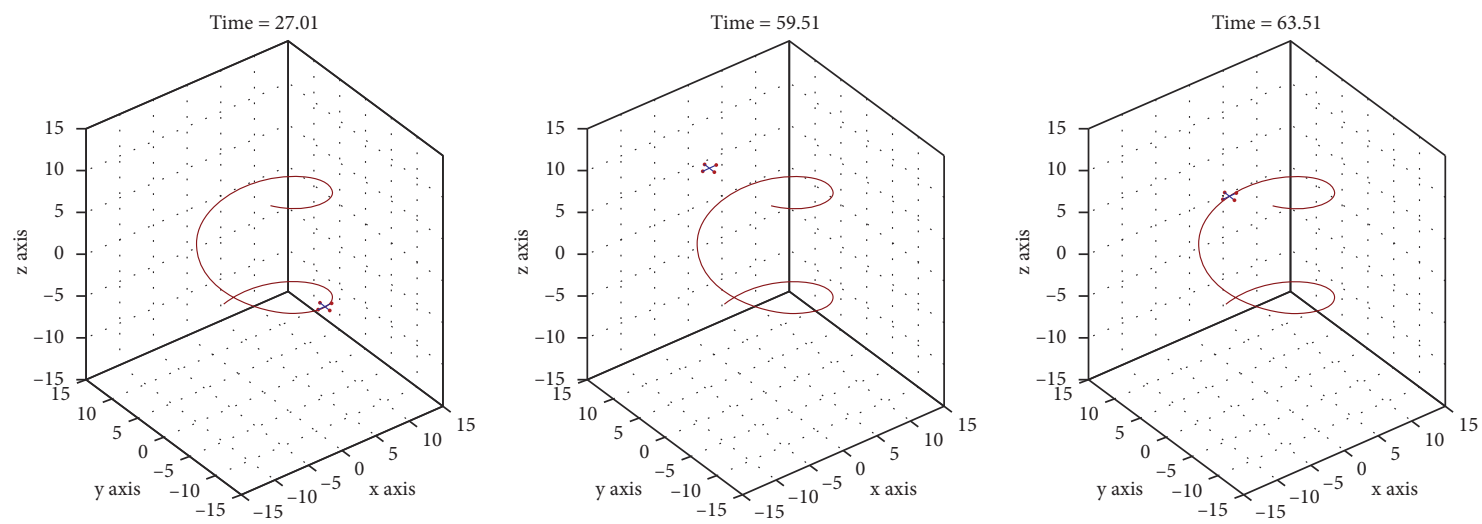

FIGURE 15: Helical trajectory tracking using fuzzy-based SMC (FSMC). 

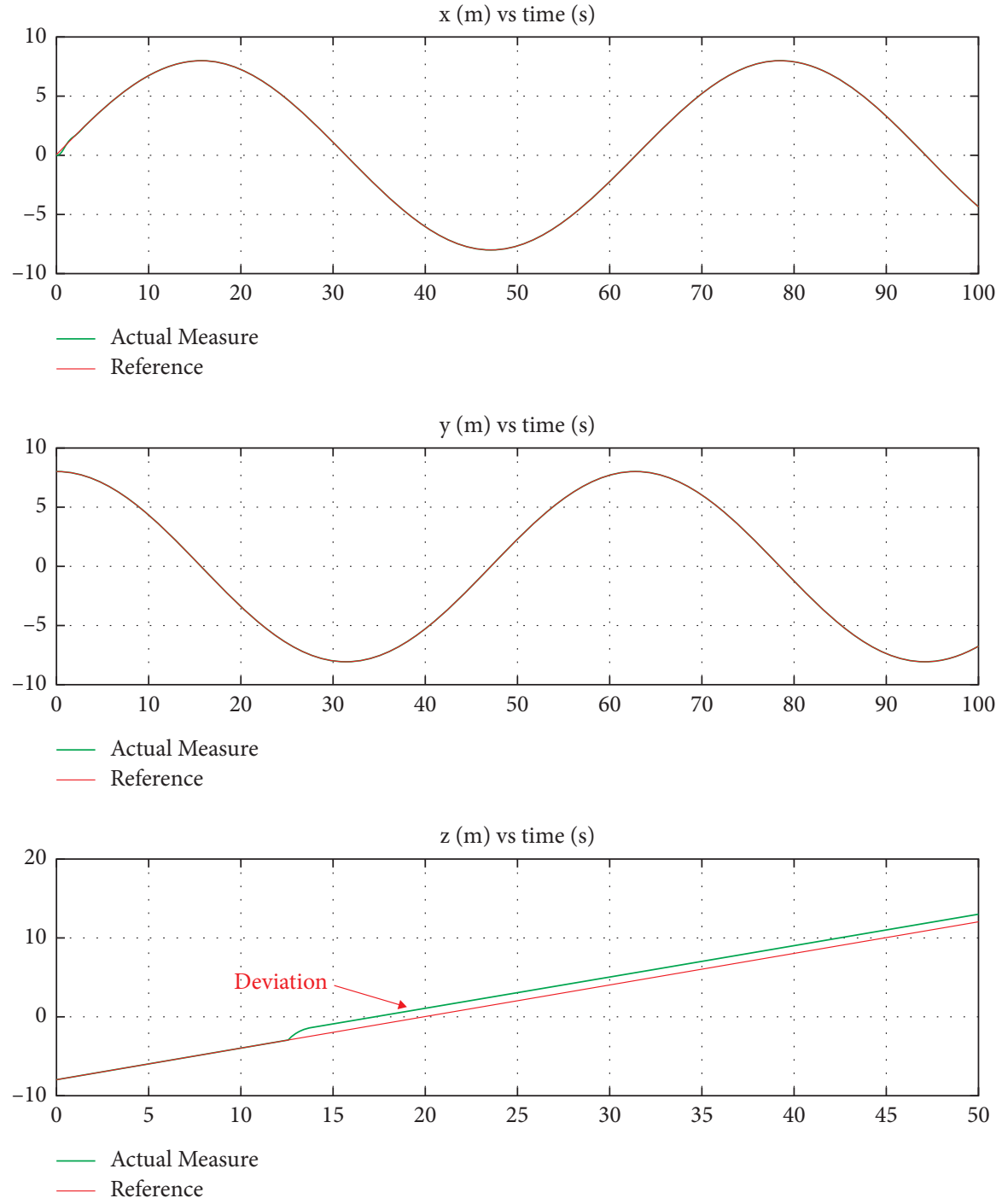

FIGURE 16: Response of positional vectors using FSMC design.
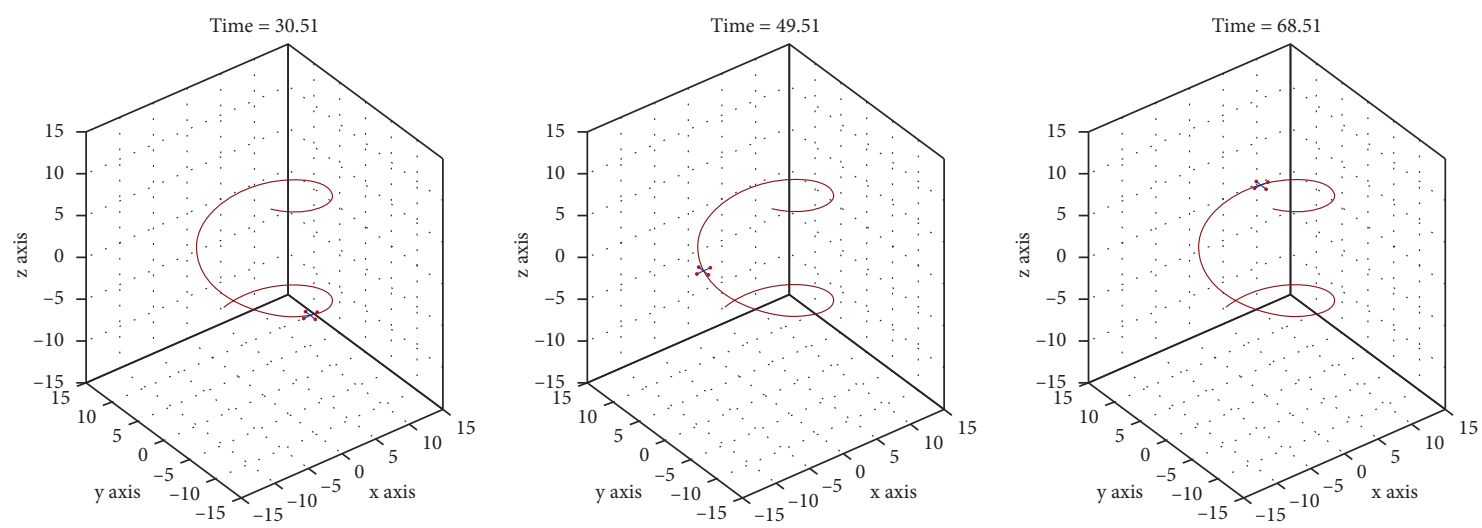

FIgUre 17: Helical trajectory tracking using DLSDF-SMC with PE and DO Design. 

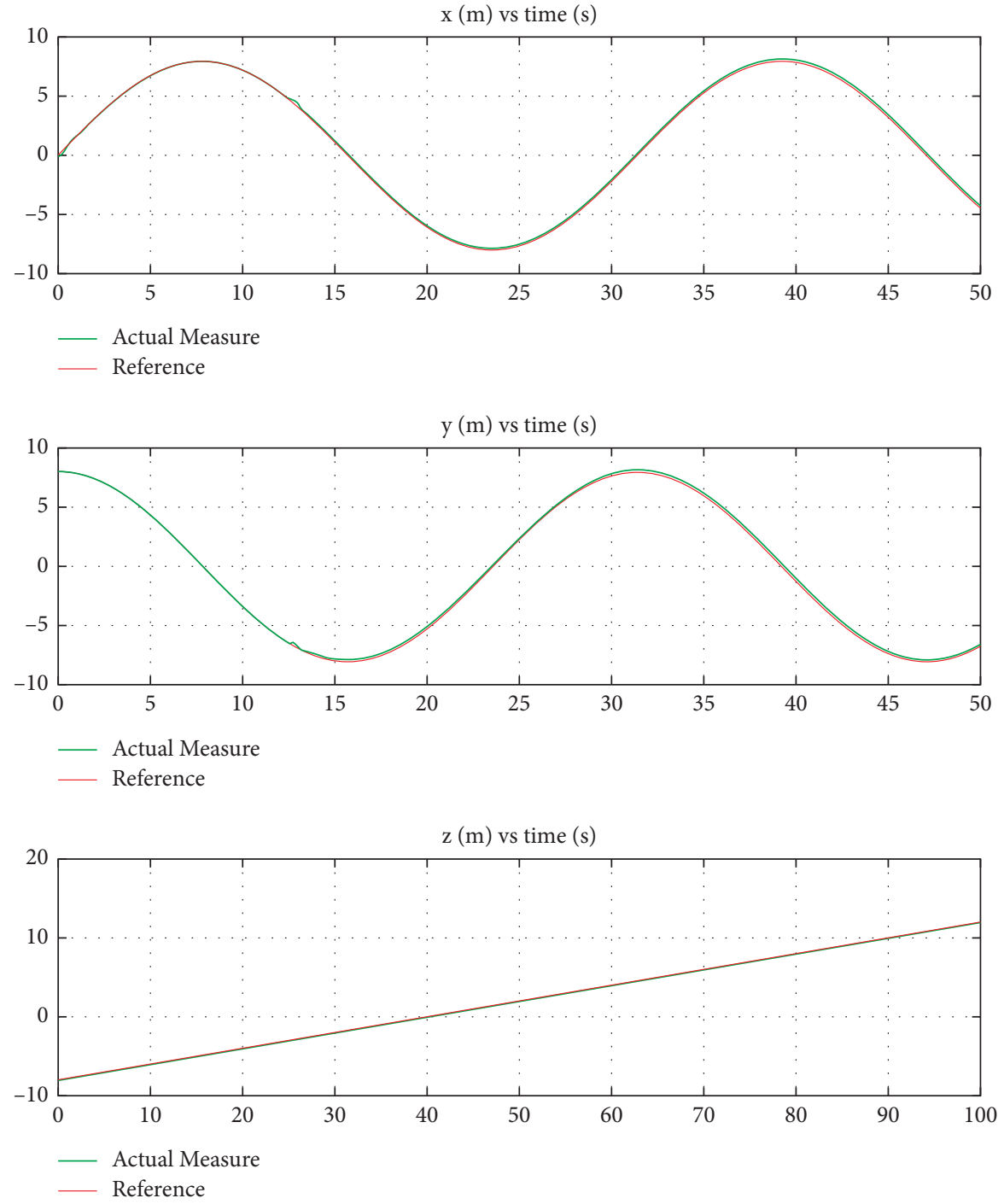

FIGURE 18: Response of positional vectors using DLSDF-SMC with PE and DO design.

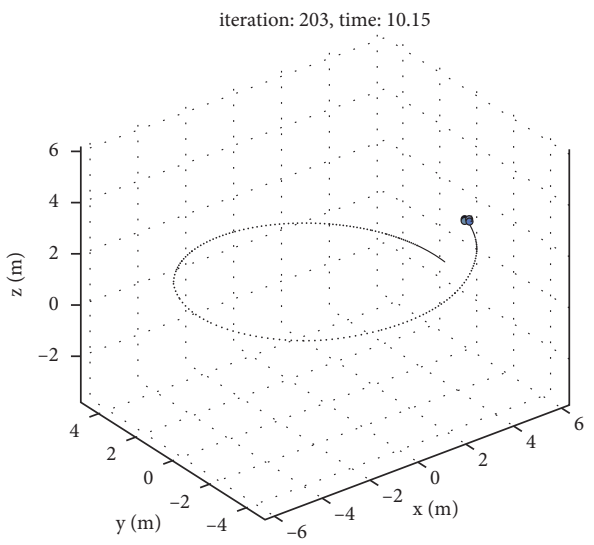

(a)

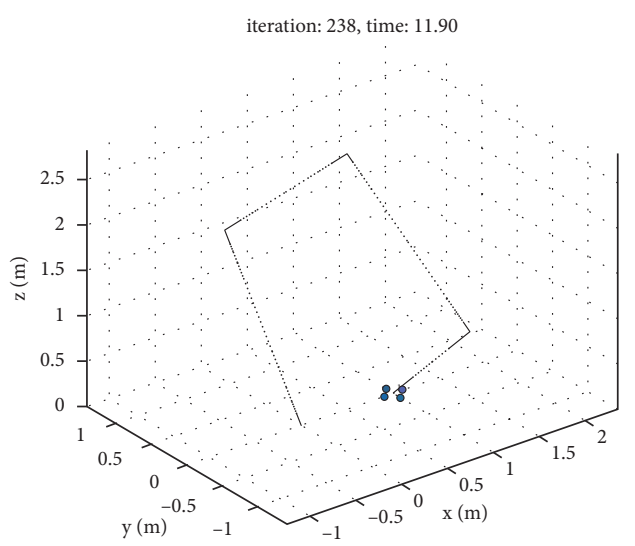

(b)

FIgURE 19: Circular and diamond-shaped trajectory tracking using DLSDF-SMC with PE and DO. 

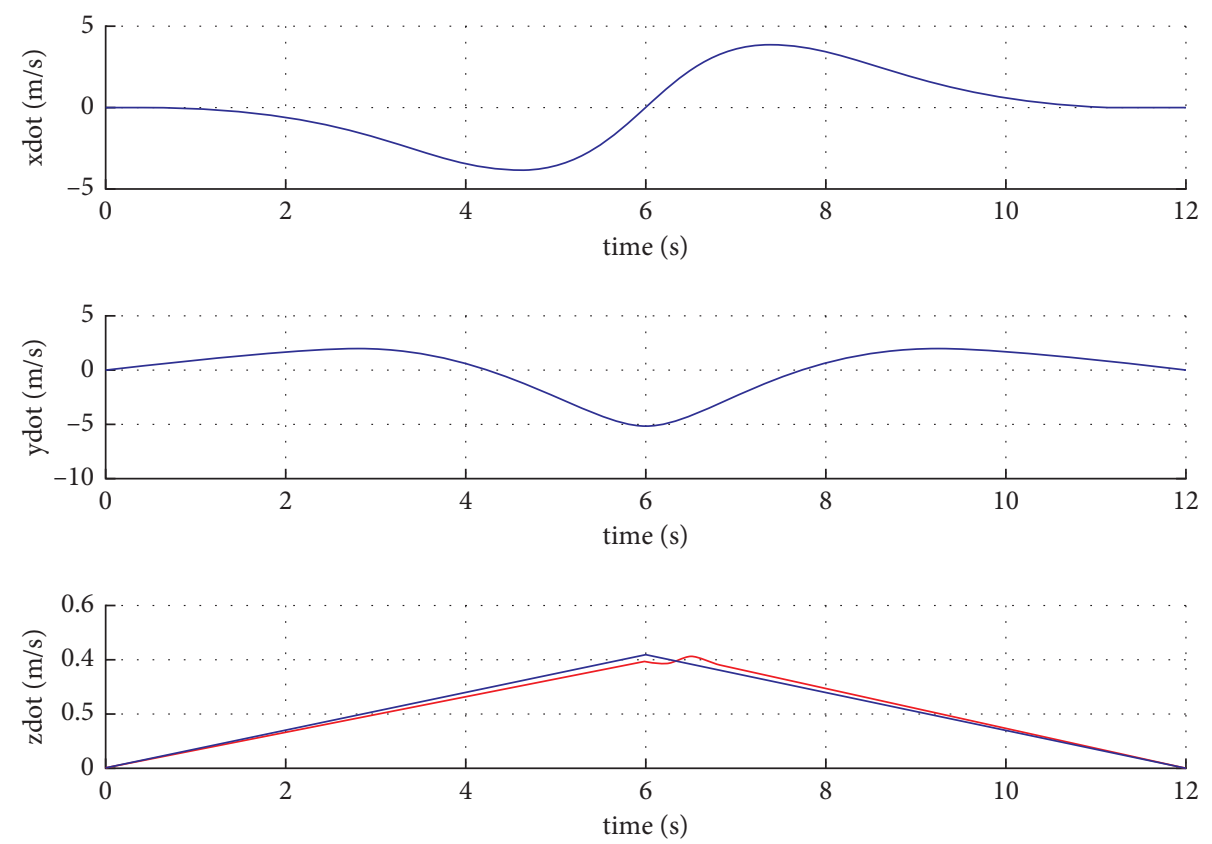

FIGURE 20: Linear velocity response while circular trajectory tracking via DLSDF-SMC with PE + DO.
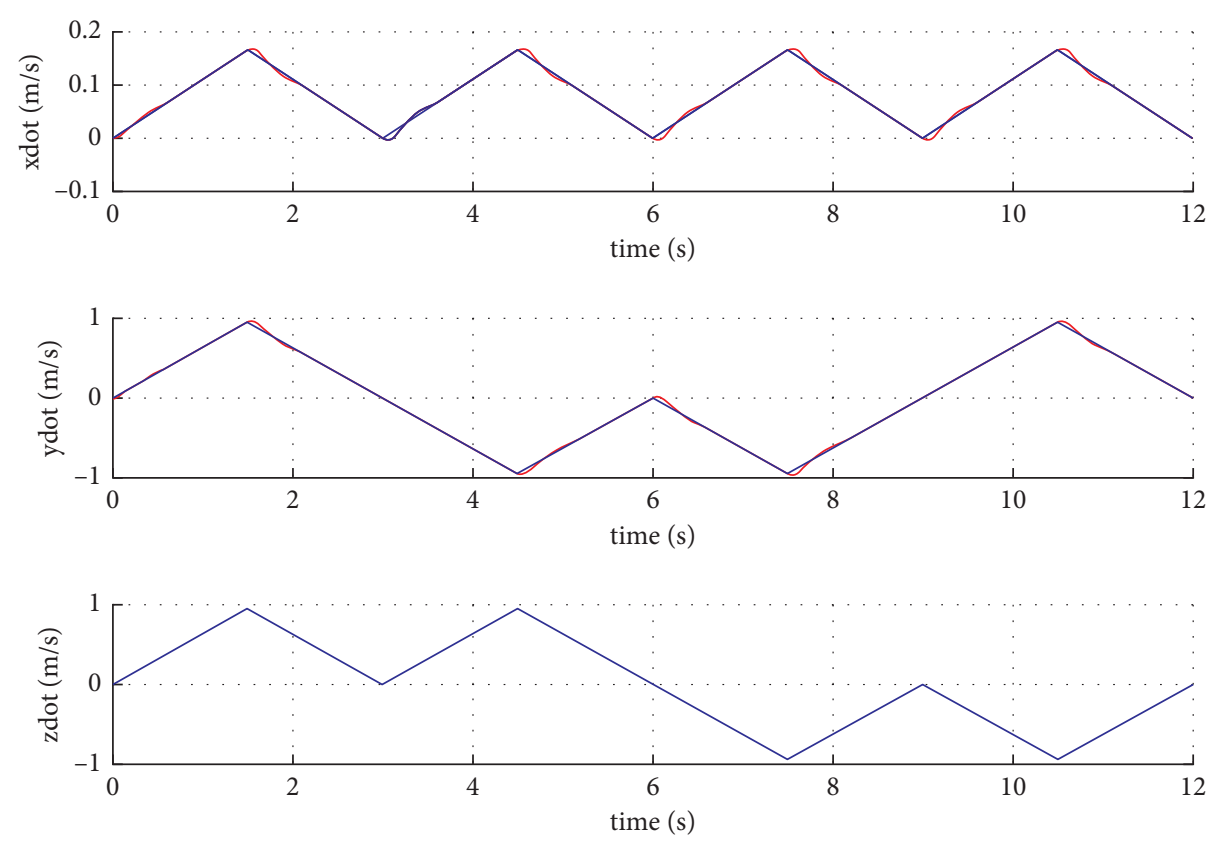

FIGURE 21: Linear velocity response while diamond-shaped trajectory tracking via DLSDF-SMC with PE + DO.

total power consumption by the four brushless DC motors. It is computed using an ordinary integral square method as shown in equation (49). In the same way, one may compute the chattering phenomena using the following equation:

$$
\operatorname{ISE}=\int_{0}^{\infty} e^{2}(t) \mathrm{d} t .
$$

Table 6 not only shares but also summarizes the overall performance of three control designs where our proposal algorithm outclasses the remaining two algorithms. These all results can also be derived using the simple command in MATLAB for the provided state-space model, that is, $\operatorname{step}(A$, $B, C, D)$, where $A, B, C$, and $D$ are the state-space matrices. However, one may see that there are also some factors that have not been compared so far. This is because, at this moment, the algorithm is tested for these parameters. The research work is in progress, and there is still a need to do some mathematical amendments. 

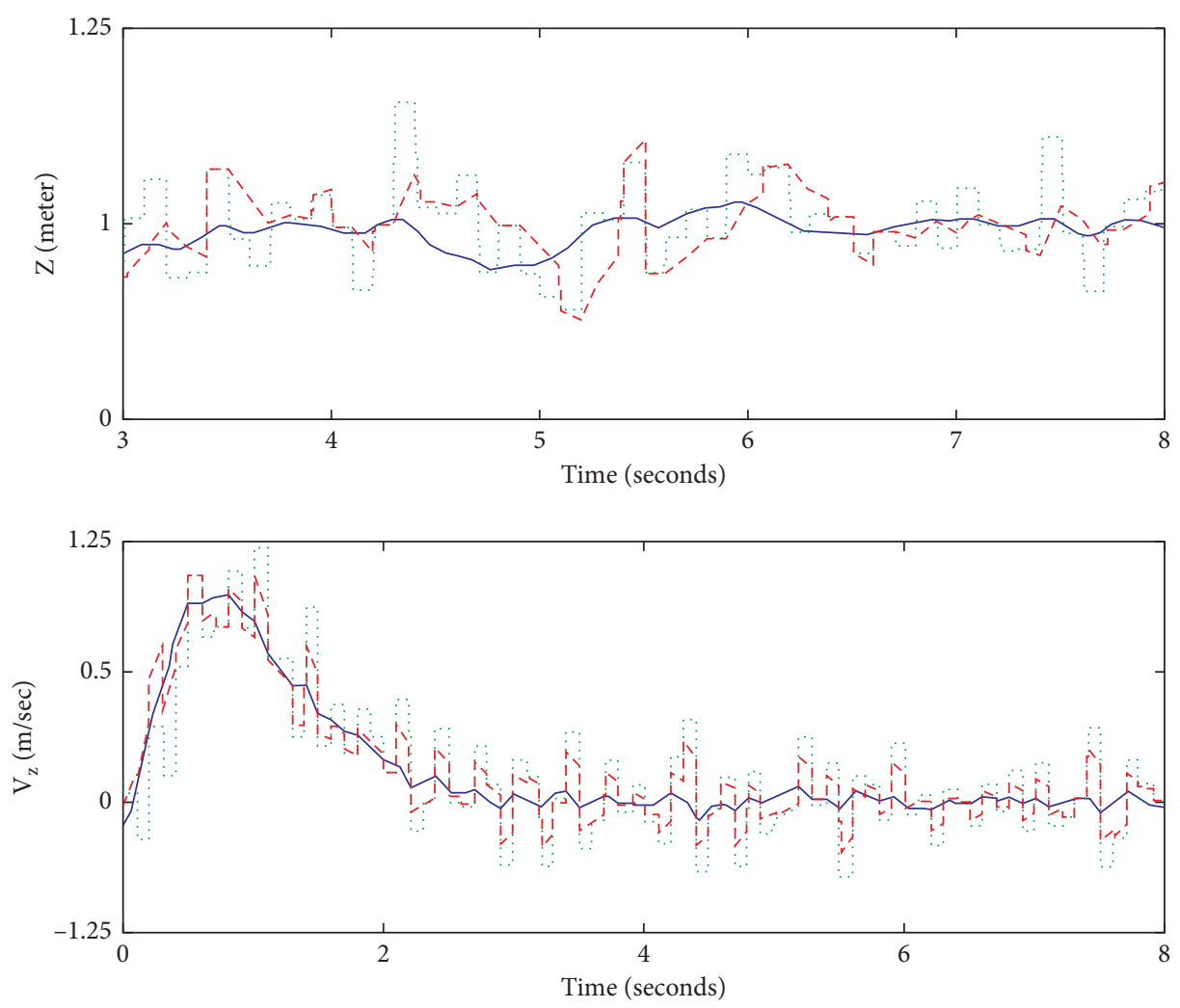

Figure 22: Performance of state estimation using PE and DO design.

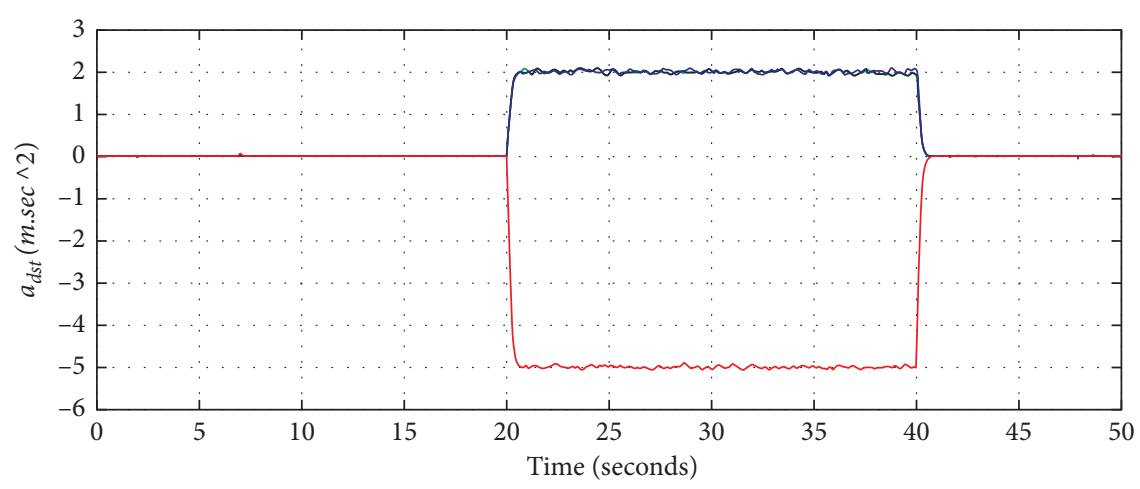

FIGURE 23: Identifying the disturbance from time instant $20^{\text {th }}$ to $40^{\text {th }}$ second.

TABle 6: Summary of SMC, FSMC, and DLSDF-SMC with PE and DO designs.

\begin{tabular}{lcccc}
\hline Control designs & Execution time (sec) & Chattering effect (\%) & Control input energy (CIE) (\%) & Steady-state error \\
\hline SMC & 100 & 4.36 & 6.54 & 4.0 \\
F-SMC & 100 & 3.275 & 6.54 & 4.0 \\
DLSDF-SMC (PE + DO) & 50 & 2.543 & 4.75 & 3.2 \\
\hline
\end{tabular}

\section{Conclusion}

The manuscript presents an underactuated quadrotor unmanned aerial vehicle (QUAV; UAV). It is underactuated because of the inequality between control inputs and the degree of freedom such that the control inputs are less than DOF. This research demonstrates the comparative simulation work between SMC, FSMC, and PIO-based DLSDF-SMC design to address the major limitations. First, this paper describes the conventional SMC control design as one of the highly sensitive control strategies for any sort of external disturbances and unmodelled dynamic factors. This is the reason behind the high number of oscillations that are experienced by four brushless DC motors of quadrotor UAV. Second, one may see fuzzy version of SMC design that reduces the chattering effect and control input energy (CIE). 
The convergence rate of all positional vectors is seen slower; thus, quadrotor craft with such rates cannot perform aggressive maneuvers. Therefore, this paper suggests dualloop single-dimension fuzzy sliding mode control technique, which is fused with position estimator and disturbance observer. This not only reduces the chattering effect but also fastens the convergence rate so that quadrotor can perform some extraordinary maneuvers. In addition to this, the steady-state error is also improvised as compared to the other two control techniques, that is, SMC and FSMC. The simulation results for helical, circular, and diamond-shaped trajectories show the robustness of the proposed algorithm. The work is still in progress to perform some more simulations to compare the remaining performance parameters, whereas this proposed algorithm is to be implemented via a specific hardware configuration to validate the software results with hardware results. [67,71-73]

\section{Data Availability}

The data used to support the findings of this study are available from the corresponding author upon request.

\section{Conflicts of Interest}

The authors declare that there are no conflicts of interest.

\section{Authors' Contributions}

Ghulam E Mustafa Abro, Vijanth Sagayan Asirvadam, Zain Anwar Ali, and Saiful Azrin Bin Mohd Zulkifli were responsible for idea and conceptualization; literature review was performed equally by all the authors; data analysis was done by Ghulam E Mustafa Abro and Saiful Azrin Bin Mohd Zulkifli; and the manuscript was drafted by Ghulam E Mustafa Abro and critically revised by Saif ul Azrin Bin Mohd Zulkifli, Zain Anwar Ali, and Vijanth Sagayan Asirvadam.

\section{Acknowledgments}

This research was funded by Yayasan Universiti Teknologi Petronas (YUTP), grant no. 015LC0-316, and the APC was funded by Research Management Centre, Universiti Teknologi Petronas, Malaysia, under the same grant. The authors wish to extend their sincere thanks to the Center of Graduate Studies, Universiti Teknologi, PETRONAS, Malaysia, for providing state-of-the-art research facilities to carry out this work. The authors are also grateful to them for providing an opportunity to work in a Graduate Research Assistantship Scheme to pursue research studies at the Department of Electrical and Electronic Engineering under Yayasan Universiti Teknologi Petronas (YUTP) Funding, grant no. 015CL0-316.

\section{References}

[1] X. Ding, X. Wang, Y. Yu, and C. Zha, "Dynamics modeling and trajectory tracking control of a quadrotor unmanned aerial vehicle (QUAV)," Journal of Dynamic Systems, Measurement, and Control, vol. 1392 pages, 2017.

[2] F. Kendoul, Z. Yu, and K. Nonami, "Guidance and nonlinear control system for autonomous flight of minirotorcraft unmanned aerial vehicles," Journal of Field Robotics, vol. 27, no. 3, pp. 311-334, 2010.

[3] A. Tayebi and S. McGilvray, "Attitude stabilization of a VTOL quadrotor aircraft," IEEE Transactions on Control Systems Technology, vol. 14, no. 3, pp. 562-571, 2006.

[4] G. E. M. Abro, V. S. Asirvadam, S. A. B. M. Zulkifli, A. Sattar, D. Kumar, and A. Anwer, "Effects of unmodelled dynamic factors on an under-actuated quadrotor: a review of hybrid observer design methods," Measurement and Control, vol. 53, no. 9-10, pp. 1978-1987, 2020.

[5] A. Isidori, L. Marconi, and A. Serrani, "Robust nonlinear motion control of a helicopter," in Robust Autonomous Guidance, pp. 149-192, Springer, London, UK, 2003.

[6] S. Islam, P. X. Liu, and A. El Saddik, "Nonlinear adaptive control for quadrotor flying vehicle," Nonlinear Dynamics, vol. 78, no. 1, pp. 117-133, 2014.

[7] H. Liu, G. Lu, and Y. Zhong, "Robust output tracking control of a laboratory helicopter for automatic landing," International Journal of Systems Science, vol. 45, no. 11, pp. 22422250, 2014.

[8] H.-N. Nguyen, C. Ha, and D. Lee, "Mechanics, control and internal dynamics of quadrotor tool operation," Automatica, vol. 61, pp. 289-301, 2015.

[9] O. Shakernia, Y. Ma, T. J. Koo, and S. Sastry, "Landing an unmanned air vehicle: vision based motion estimation and nonlinear control," Asian Journal of Control, vol. 1, no. 3, pp. 128-145, 1999.

[10] A. Israr, G. E. Mustafa Abro, M. Sadiq Ali Khan, M. Farhan, B. Mohd Zulkifli, and S. Ul Azrin, "Internet of things (IoT)Enabled unmanned aerial vehicles for the inspection of construction sites: a vision and future directions," Mathematical Problems in Engineering, vol. 2021, Article ID 9931112, 15 pages, 2021.

[11] J. Li and Y. Li, "Dynamic analysis and PID control for a quadrotor," in Proceedings of the 2011 IEEE International Conference on Mechatronics and Automation, pp. 573-578, IEEE, Beijing, China, August 2011.

[12] H. Bolandi, M. Rezaei, R. Mohsenipour, H. Nemati, and S. M. Smailzadeh, "Attitude control of a quadrotor with optimized PID controller," Intelligent Control and Automation, vol. 4, no. 3, pp. 342-349, 2013.

[13] G. V. Raffo, M. G. Ortega, and F. R. Rubio, "An integral predictive/nonlinear $H_{\infty}$ control structure for a quadrotor helicopter," Automatica, vol. 46, no. 1, pp. 29-39, 2010.

[14] F. Chen, R. Jiang, K. Zhang, B. Jiang, and G. Tao, "Robust backstepping sliding-mode control and observer-based fault estimation for a quadrotor UAV," IEEE Transactions on Industrial Electronics, vol. 63, no. 8, pp. 5044-5056, 2016.

[15] Z. T. Dydek, A. M. Annaswamy, and E. Lavretsky, "Adaptive control of quadrotor UAVs: a design trade study with flight evaluations," IEEE Transactions on Control Systems Technology, vol. 21, no. 4, pp. 1400-1406, 2012.

[16] T. Dierks and S. Jagannathan, "Output feedback control of a quadrotor UAV using neural networks," IEEE Transactions on Neural Networks, vol. 21, no. 1, pp. 50-66, 2009.

[17] D. Ma, Y. Xia, T. Li, and K. Chang, "Active disturbance rejection and predictive control strategy for a quadrotor helicopter," IET Control Theory \& Applications, vol. 10, no. 17, pp. 2213-2222, 2016. 
[18] X. Shao, L. Wang, J. Li, and J. Liu, "High-order ESO based output feedback dynamic surface control for quadrotors under position constraints and uncertainties," Aerospace Science and Technology, vol. 89, pp. 288-298, 2019.

[19] M. Fliess, J. Lévine, P. Martin, and P. Rouchon, "Flatness and defect of non-linear systems: introductory theory and examples," International Journal of Control, vol. 61, no. 6, pp. 1327-1361, 1995.

[20] H. Sira-Ramírez, A. Luviano-Juarez, and J. Cortés-Romero, "Flatness-based linear output feedback control for disturbance rejection and tracking tasks on a Chua's circuit," International Journal of Control, vol. 85, no. 5, pp. 594-602, 2012.

[21] Y. Xia, F. Pu, S. Li, and Y. Gao, "Lateral path tracking control of autonomous land vehicle based on ADRC and differential flatness," IEEE Transactions on Industrial Electronics, vol. 63, no. 5, pp. 3091-3099, 2016.

[22] J. F. Guerrero-Castellanos, H. Rifaï, V. Arnez-Paniagua, J. Linares-Flores, L. Saynes-Torres, and S. Mohammed, "Robust active disturbance rejection control via control lyapunov functions: application to actuated-ankle-foot-orthosis," Control Engineering Practice, vol. 80, pp. 49-60, 2018.

[23] M. Ramirez-Neria, Z. Gao, H. Sira-Ramirez, R. GarridoMoctezuma, and A. Luviano-Juarez, "Trajectory tracking for an inverted pendulum on a cart: an active disturbance rejection control approach," in Proceedings of the 2018 Annual American Control Conference (ACC), pp. 4881-4886, IEEE, Milwaukee, WI, USA, June 2018.

[24] H. Sira-Ramírez, A. Luviano-Juárez, M. Ramírez-Neria, and E. W. Zurita-Bustamante, Active Disturbance Rejection Control of Dynamic Systems: A Flatness Based Approach, Butterworth-Heinemann, Oxford, UK, 2018.

[25] G. G. Rigatos, "Non-linear feedback control of the p 53 protein- mdm 2 inhibitor system using the derivative-free non-linear Kalman filter," IET Systems Biology, vol. 10, no. 3, pp. 94-106, 2016.

[26] G. Rigatos, G. Raffo, and P. Siano, "AUV control and navigation with differential flatness theory and derivative-free nonlinear Kalman filtering," Intelligent Industrial Systems, vol. 3, no. 1, pp. 29-41, 2017.

[27] E.-H. Zheng, J.-J. Xiong, and J.-L. Luo, "Second order sliding mode control for a quadrotor UAV," ISA Transactions, vol. 53, no. 4, pp. 1350-1356, 2014.

[28] Z. Fang, Z. Zhang, J. Liang, and J. Wang, "Feedback linearization and continuous sliding mode control for a quadrotor UAV," in Proceedings of the 2008 27th Chinese Control Conference, pp. 349-353, IEEE, Kunming, China, July 2008.

[29] Z. Gao, "Scaling and bandwidth-parameterization based controller tuning," Proceedings-American Control Conference, vol. 6, pp. 4989-4996, 2006.

[30] X. Yang and Y. Huang, "Capabilities of extended state observer for estimating uncertainties," in Proceedings of the 2009 American Control Conference, pp. 3700-3705, IEEE, St. Louis, MI, USA, June 2009.

[31] Y. Zhang, Z. Jiang, H. Yang, J. Cheng, and W. Zhang, "Highorder extended state observer-enhanced control for a hypersonic flight vehicle with parameter uncertainty and external disturbance," Proceedings of the Institution of Mechanical Engineers-Part G: Journal of Aerospace Engineering, vol. 229, no. 13, pp. 2481-2496, 2015.

[32] N. Fethalla, M. Saad, H. Michalska, and J. Ghommam, "Robust observer-based dynamic sliding mode controller for a quadrotor UAV," IEEE access, vol. 6, pp. 45846-45859, 2018.
[33] Y. Huang, Z. Zheng, L. Sun, and M. Zhu, "Saturated adaptive sliding mode control for autonomous vessel landing of a quadrotor," IET Control Theory \& Applications, vol. 12, no. 13, pp. 1830-1842, 2018.

[34] B. Mu, K. Zhang, and Y. Shi, "Integral sliding mode flight controller design for a quadrotor and the application in a heterogeneous multi-agent system," IEEE Transactions on Industrial Electronics, vol. 64, no. 12, pp. 9389-9398, 2017.

[35] T. Tomić, "Evaluation of acceleration-based disturbance observation for multicopter control," in Proceedings of the 2014 European Control Conference (ECC), pp. 2937-2944, IEEE, Strasbourg, France, June 2014.

[36] A. Zulu and S. John, "A review of control algorithms for autonomous quadrotors," 2016, https://arxiv.org/abs/1602. 02622 .

[37] G. E. M. Abro, V. S. Asirvadam, and S. A. Zulkifli, "SingleInput fuzzy-sliding mode control for an underactuated quadrotor craft," in Proceedings of the 2020 IEEE 2nd International Conference on Artificial Intelligence in Engineering and Technology (IICAIET), pp. 1-6, IEEE, Kota Kinabalu, Malaysia, September 2020.

[38] M. Cui, W. Liu, H. Liu, H. Jiang, and Z. Wang, "Extended state observer-based adaptive sliding mode control of differentialdriving mobile robot with uncertainties," Nonlinear Dynamics, vol. 83, no. 1-2, pp. 667-683, 2016.

[39] H. Duan and C. Li, "Quantum-behaved brain storm optimization approach to solving Loney's solenoid problem," IEEE Transactions on Magnetics, vol. 51, no. 1, pp. 1-7, 2014.

[40] H. B. Duan and S. Q. Liu, "Non-linear dual-mode receding horizon control for multiple unmanned air vehicles formation flight based on chaotic particle swarm optimisation," IET Control Theory \& Applications, vol. 4, no. 11, pp. 2565-2578, 2010.

[41] H. Duan, Q. Luo, Y. Shi, and G. Ma, "?Hybrid particle swarm optimization and genetic algorithm for multi-UAV formation reconfiguration," IEEE Computational Intelligence Magazine, vol. 8, no. 3, pp. 16-27, 2013.

[42] S. J. Lee, I. Jang, and H. J. Kim, "Fail-Safe flight of a fullyactuated quadrotor in a single motor failure," IEEE Robotics and Automation Letters, vol. 5, no. 4, pp. 6403-6410, 2020.

[43] P. Manouchehri, R. Ghasemi, and A. Toloei, "Distributed fuzzy adaptive sliding mode formation for nonlinear multiquadrotor systems," International Journal of Engineering, vol. 33, no. 5, pp. 798-804, 2020.

[44] O. D. Dantsker, M. Caccamo, M. Vahora, and R. Mancuso, "Flight \& ground testing data set for an unmanned aircraft: great planes avistar elite," in AIAA Scitech 2020 Forump. 780, American Institute of Aeronautics and Astronautics, Orlando, FL, USA, 2020.

[45] B. Wu, X.-H. Chang, and X. Zhao, "Fuzzy $H_{\infty}$ output feedback control for nonlinear NCSs with quantization and stochastic communication protocol," IEEE Transactions on Fuzzy Systems, vol. 29, no. 9, pp. 2623-2634, 2021.

[46] X. Song, M. Wang, C. K. Ahn, and S. Song, "Finite-time fuzzy bounded control for semilinear PDE systems with quantized measurements and markov jump actuator failures," IEEE Transactions on Cybernetics, 2021.

[47] A. Bagheri, T. Karimi, and N. Amanifard, "Tracking performance control of a cable communicated underwater vehicle using adaptive neural network controllers," Applied Soft Computing, vol. 10, no. 3, pp. 908-918, 2010.

[48] K. Wang, C. Hua, J. Chen, and M. Cai, "Dual-loop integral sliding mode control for robust trajectory tracking of a 
quadrotor," International Journal of Systems Science, vol. 51, no. 2, pp. 203-216, 2020.

[49] Y. Wang, H. Cai, J. Zhang, and X. Li, "Disturbance attenuation predictive optimal control for quad-rotor transporting unknown varying payload," IEEE Access, vol. 8, pp. 44671-44686, 2020.

[50] J. Sanwale, P. Trivedi, M. Kothari, and A. Malagaudanavar, "Quaternion-based position control of a quadrotor unmanned aerial vehicle using robust nonlinear third-order sliding mode control with disturbance cancellation," Proceedings of the Institution of Mechanical Engineers-Part G: Journal of Aerospace Engineering, vol. 234, no. 4, pp. 997$1013,2020$.

[51] O. Mofid and S. Mobayen, "Adaptive finite-time back-stepping global sliding mode tracker of quad-rotor UAVs under model uncertainty, wind perturbation and input saturation," IEEE Transactions on Aerospace and Electronic Systems, 2021.

[52] O. Mofid, S. Mobayen, C. Zhang, and B. Esakki, "Desired tracking of delayed quadrotor UAV under model uncertainty and wind disturbance using adaptive super-twisting terminal sliding mode control," ISA Transactions, 2021.

[53] O. Mofid, S. Mobayen, and W.-K. Wong, "Adaptive terminal sliding mode control for attitude and position tracking control of quadrotor UAVs in the existence of external disturbance," IEEE Access, vol. 9, pp. 3428-3440, 2020.

[54] K. N. Tiwari, L. M. Waghmare, and P. Krishnankutty, "Single input fuzzy logic controller tuning for steering control of autonomous underwater vehicle: genetic algorithm approach," in Proceedings of the 2016 Indian Control Conference (ICC), pp. 335-340, IEEE, Hyderabad, India, January 2016.

[55] M. A. Ahmad, M. S. Saealal, R. M. T. Raja Ismail, M. A. Zawawi, A. N. K. Nasir, and M. S. Ramli, "Single input fuzzy controller with command shaping schemes for doublependulum-type overhead crane," American Institute of Physics, vol. 1337, no. 1, pp. 113-117, 2011.

[56] K. Ishaque, S. S. Abdullah, S. M. Ayob, and Z. Salam, "A simplified approach to design fuzzy logic controller for an underwater vehicle," Ocean Engineering, vol. 38, no. 1, pp. 271-284, 2011.

[57] M. Faisal Farhan, N. S. Abdul Shukor, M. Ashraf Ahmad, M. Helmi Suid, M. Riduwan Ghazali, and M. F. Mat Jusof, "A simplify fuzzy logic controller design based safe experimentation dynamics for pantograph-catenary system," Indonesian Journal of Electrical Engineering and Computer Science, vol. 14, no. 2, pp. 903-911, 2019.

[58] B. J. Emran and H. Najjaran, "A review of quadrotor: an underactuated mechanical system," Annual Reviews in Control, vol. 46, pp. 165-180, 2018.

[59] M. Asif, A. Y. Memon, and M. Junaid Khan, "Output feedback control for trajectory tracking of wheeled mobile robot," Intelligent Automation \& Soft Computing, vol. 22, no. 1, pp. 75-87, 2016.

[60] G. E. M. Abro, B. Jabeen, and A. Manan, "Stabilization of nonholonomic 03 DOF hovercraft using robust RST control design," Sukkur IBA Journal of Emerging Technologies, vol. 2, no. 1, pp. 45-50, 2019.

[61] L. Besnard, Y. B. Shtessel, and B. Landrum, "Quadrotor vehicle control via sliding mode controller driven by sliding mode disturbance observer," Journal of the Franklin Institute, vol. 349, no. 2, pp. 658-684, 2012.

[62] H. Bouadi, S. S. Cunha, D. Antoine, and F. Mora-Camino, "Adaptive sliding mode control for quadrotor attitude stabilization and altitude tracking," in Proceedings of the 2011 IEEE 12th International Symposium on Computational
Intelligence and Informatics (CINTI), pp. 449-455, IEEE, Budapest, Hungary, November 2011.

[63] F. Sharifi, M. Mirzaei, B. W. Gordon, and Y. Zhang, "Fault tolerant control of a quadrotor UAV using sliding mode control," in Proceedings of the 2010 Conference on Control and Fault-Tolerant Systems (SysTol), pp. 239-244, IEEE, Nice, France, October 2010.

[64] H. Liu, J. Xi, and Y. Zhong, "Robust attitude stabilization for nonlinear quadrotor systems with uncertainties and delays," IEEE Transactions on Industrial Electronics, vol. 64, no. 7, pp. 5585-5594, 2017.

[65] K. Li, Z. Li, and H. Liu, "Robust control for hypersonic vehicles with multiple uncertainties and time-varying delays," in Proceedings of the 2016 Chinese Control and Decision Conference (CCDC), pp. 2491-2496, IEEE, Yinchuan, China, May 2016.

[66] B. Mu, Y. Pei, and Y. Shi, "Integral sliding mode control for a quadrotor in the presence of model uncertainties and external disturbances," in Proceedings of the 2017 American Control Conference (ACC), pp. 5818-5823, IEEE, Seattle, WA, USA, May 2017.

[67] G. E. M. Abro, S. A. B. M. Zulkifli, V. S. Asirvadam, and Z. A. Ali, "Model-free-based single-dimension fuzzy SMC design for underactuated quadrotor UAV," Actuators, vol. 10, no. 8, p. 191, 2021.

[68] H. Duan and P. Qiao, "Pigeon-inspired optimization: a new swarm intelligence optimizer for air robot path planning," International Journal of Intelligent Computing and Cybernetics, vol. 7, no. 1, pp. 24-37, 2014.

[69] H. B. Duan, H. Qiu, and Y. M. Fan, "Unmanned aerial vehicle close formation cooperative control based on predatory escaping pigeon-inspired optimization," Scientia Sinica Technologica, vol. 45, no. 6, pp. 559-572, 2015.

[70] J.-J. Xiong and G.-B. Zhang, "Global fast dynamic terminal sliding mode control for a quadrotor UAV," ISA Transactions, vol. 66, pp. 233-240, 2017.

[71] L. A. Zadeh, Fuzzy Logic Toolbox for Use with Matlab, The MathWorks Inc., Portola Valley, CA, USA, 1999.

[72] X. Wang, B. Shirinzadeh, and M. H. Ang, "Nonlinear doubleintegral observer and application to quadrotor aircraft," IEEE Transactions on Industrial Electronics, vol. 62, no. 2, pp. 1189-1200, 2014.

[73] Y. Qi, Y. Zhu, J. Wang, J. Shan, and H. H. T. Liu, "MUDEbased control of quadrotor for accurate attitude tracking," Control Engineering Practice, vol. 108, Article ID 104721, 2021.

[74] D. D. Dhadekar, P. D. Sanghani, K. K. Mangrulkar, and S. E. Talole, "Robust control of quadrotor using uncertainty and disturbance estimation," Journal of Intelligent and Robotic Systems, vol. 101, no. 3, pp. 1-21, 2021.

[75] A. Castillo, R. Sanz, P. Garcia, W. Qiu, H. Wang, and C. Xu, "Disturbance observer-based quadrotor attitude tracking control for aggressive maneuvers," Control Engineering Practice, vol. 82, pp. 14-23, 2019.

[76] R. Sanz, P. Garcia, Q.-C. Zhong, and A. Pedro, "Robust control of quadrotors based on an uncertainty and disturbance estimator," Journal of Dynamic Systems, Measurement, and Control, vol. 138, no. 7, 2016.

[77] J. Yao, J. Zongxia Jiao, and M. Dawei, "Adaptive robust control of DC motors with extended state observer," IEEE Transactions on Industrial Electronics, vol. 61, no. 7, pp. 3630-3637, 2013. 